\title{
Characterization of multi-scale ionospheric irregularities using ground-based and space-based GNSS observations
}

\author{
YuXiang Peng ${ }^{1,2,5^{*}} \mathbb{B}$, Wayne A Scales ${ }^{1,2}$, Michael D Hartinger ${ }^{3}$, Zhonghua $\mathrm{Xu}^{1,2,4}$ and Shane Coyle $\mathrm{e}^{1,2}$
}

\begin{abstract}
Ionospheric irregularities can adversely affect the performance of Global Navigation Satellite System (GNSS). However, this opens the possibility of using GNSS as an effective ionospheric remote sensing tool. Despite ionospheric monitoring has been undertaken for decades, these irregularities in multiple spatial and temporal scales are still not fully understood. This paper reviews Virginia Tech's recent studies on multi-scale ionospheric irregularities using ground-based and space-based GNSS observations. First, the relevant background of ionospheric irregularities and their impact on GNSS signals is reviewed. Next, three topics of ground-based observations of ionospheric irregularities for which GNSS and other ground-based techniques are used simultaneously are reviewed. Both passive and active measurements in high-latitude regions are covered. Modelling and observations in mid-latitude regions are considered as well. Emphasis is placed on the increased capability of assessing the multi-scale nature of ionospheric irregularities using other traditional techniques (e.g., radar, magnetometer, high frequency receivers) as well as GNSS observations (e.g., Total-Electron-Content or TEC, scintillation). Besides ground-based observations, recent advances in GNSS space-based ionospheric measurements are briefly reviewed. Finally, a new space-based ionospheric observation technique using GNSS-based spacecraft formation flying and a differential TEC method is demonstrated using the newly developed Virginia Tech Formation Flying Testbed (VTFFTB). Based on multi-constellation multi-band GNSS, the VTFFTB has been developed into a hardware-in-the-loop simulation testbed with external high-fidelity global ionospheric model(s) for 3-satellite formation flying, which can potentially be used for new multi-scale ionospheric measurement mission design.
\end{abstract}

Keywords: GNSS, lonospheric irregularities, Remote sensing, Active experiment, Spacecraft formation flying

\section{Introduction}

Ionospheric irregularities are associated with the plasma density structures in the ionosphere and can severely impact the performance of various modern technologies such as satellite communication and Global Navigation Satellite System (GNSS) Kintner et al. (2007). When GNSS Radio-Frequency (RF) signals encounter ionospheric irregularities, effects like signal delay, scintillation

\footnotetext{
*Correspondence: yuxiang7@vt.edu

${ }^{5}$ Center for Space Science and Engineering Research, Virginia Tech,

Blacksburg, VA 24061, USA

Full list of author information is available at the end of the article
}

(phase and intensity fluctuations), cycle slips, or Faraday rotation can be induced. From the perspective of GNSS applications, the positioning accuracy, precision and integrity may be reduced. Positioning accuracy refers to a time average offset between positioning solutions and true positions. While positioning precision refers to a standard deviation of the offsets between positioning solutions and true positions. In severe scintillation cases, the GNSS observability might be lost (e.g., loss-oflock) due to strong signal interruptions prior to receiver antenna reception. These irregularity impacts can increase the dilution of position and degrade the Kalman filter's estimation performance. The space weather 
impacts on GNSS applications were not fully appreciated, until recently when more and more mass-market GNSS products (e.g., high-level autonomous vehicles, mobile and Internet-of-Things devices) featuring high-precision positioning techniques (e.g., Precise Point Positioning or PPP, Real-Time Kinematic or RTK) became available. Under the effects of low-latitude ionospheric scintillation, Luo et al. (2018) observed the residuals of the BeiDou Navigation Satellite System (BDS) dual-frequency PPP can be up to $7.096 \mathrm{~m}$ in code-phase and $0.469 \mathrm{~m}$ in carrier-phase. The three-dimensional root-mean-square of BDS PPP under scintillation can be 12 times larger than that in non-scintillation conditions. More details of ionospheric irregularity and scintillation effects on GNSS positioning and navigation are reviewed in Luo et al. (2020).

Depending on the type and location, the scale size of ionospheric irregularities can range from sub-meters (Kelley 2009) to thousands of kilometers (e.g., super plasma bubbles) (Fejer and Kelley 1980). Due to nonlinear turbulent cascade and other nonlinear wave mixing processes, irregularities seeded at one spatial scale may evolve to exist over a broad range of smaller spatial scales. Therefore, irregularities originally generated at one spatial scale may ultimately be sensed by ground-based diagnostics over a range of smaller spatial scales. Possible driving mechanism of ionospheric irregularities include but not limited to geomagnetic storms (commonly known as solar storms) (Parkinson et al. 1996), plasma instabilities, e.g., Rayleigh-Taylor instability (Eltrass and Scales 2014; de Larquier et al. 2014; Eltrass et al. 2014, 2016), and gravity waves (Hooke 1968). The associated effects of solar storms on GNSS signals (measured by ground-based Global Positioning System or GPS receivers) and geomagnetic field (measured by ground-based magnetometers) will be discussed in Section Passive measurements of high-latitude ionospheric structure.

Irregularities often believed to be associated with GNSS scintillations exist in the Fresnel-scale range of 100's of meters or so (Kintner et al. 2007). However, the irregularities of observational signatures that can be sensed at smaller spatial scales typically thought not to be associated with GNSS scintillations, may have important consequences in GNSS scintillation production. During active space experiments in which high power HighFrequency (HF) radio waves interact with the ionosphere, the irregularities of decimeter $(10 \mathrm{~cm})$ scale are generated to produce GNSS phase scintillations since the GNSS wavelength is of the decimeter scale. This is considered in Section Active measurement of high-latitude ionospheric structure and impacts on GNSS. Another example recently considered is HF space weather radars that sense decameter scale irregularities, not typically thought to be associated with Fresnel-scale GNSS scintillations. These may provide a significant insight into the mechanisms producing GNSS scintillations. This is discussed in Section Modelling and observation of mid-latitude ionospheric irregularity effects on GNSS.

Due to the remote observation nature, ground-based observational methods such as GPS receivers, magnetometers, radars, and ionosondes, cannot fully cover the global ionosphere. Space-based observation techniques such as sounding rockets or satellites can be better suited to closely measuring ionospheric irregularity. Space-based GNSS measurement technique and a new ionospheric mission incubating platform will be reviewed in Section A new space-based observation technique.

\section{Ground-based GNSS remote sensing}

A great number of ground-based receivers have been deployed in different regions around the world to detect and measure ionospheric space weather including the plasma irregularities that disturb GNSS signals. The typical examples include the global Total Electron Content (TEC) maps in Madrigal database, produced by the Massachusetts Institute of Technology (MIT) (Vierinen et al. 2016) and the National Aeronautics and Space Administration (NASA) Jet Propulsion Laboratory (JPL) global TEC map (Pi et al. 1997). In this section we present a few examples of passive and active GNSS remote sensing studies. These measurements are made simultaneously with other techniques (radar, magnetometer, HF receivers, etc.). These simultaneous measurements facilitate remote sensing possibilities with enhanced multi-scale observation capabilities.

\section{Passive measurements of high-latitude ionospheric structure}

On the East Antarctic Plateau, a chain of Autonomous Adaptive Low-Power Instrument Platforms (AAL-PIP) had been established on the ice sheet along the $40^{\circ}$ magnetic meridian to observe ionospheric activity in the South Polar region (Clauer et al. 2014; Xu et al. 2019). The AAL-PIPs comprise an array of four CASES dualfrequency GPS receivers (developed by ASTRA, LLC ASTRA, http://www.astraspace.net/) with Antcom GPS antennas. Other scientific instruments include a fluxgate magnetometer and a search-coil (i.e., induction) magnetometer. They were deployed together with each CASES GNSS receiver currently at four AAL-PIP stations (namely PG2, PG3, PG4, and PG5) for space weather observations. An overview on the project motivation, system design, deployment, and GPS operation protocols of the AAl-PIP will be given in Section System overview and GPS receiver operation. The data processing of AALPIP will be explained in Section GPS data processing. The 
AAL-PIP GPS receiver chain has been used to observe ionospheric irregularities (that led to GPS scintillation), as well as to capture Ultra Low Frequency (ULF) waves (that led to geomagnetic pulsations of GPS TEC) associated with geomagnetic storms (Kim et al. 2014; Xu et al. 2019). These studies will be highlighted in Section Space weather observations.

\section{System overview and GPS receiver operation}

The high-latitude (or polar) ionosphere is the region above $60^{\circ}$ magnetic latitude (e.g., auroral zone, polar cap), where plasma instabilities and other dynamic processes (e.g., coupling physics between solar wind, magnetosphere, ionosphere and thermosphere) cause ionospheric structures and irregularities (Kelley 2009). Additionally, space weather phenomena such as aurora, plasma waves and turbulent flows often occur in these regions (Xu et al. 2017). Because of the uniqueness and complexity of the space weather in high-latitude regions, it is pivotal to monitor and study the geo-space environment in these regions. Comparably, the northern polar region is more instrumented than the southern polar region. A chain of magnetometer ground stations was deployed (then operated by the Danish National Space Institute at the Technical University of Denmark) along the western coast of Greenland. Later, the development and deployment of AAL-PIPs at the magnetic conjugate points of these Greenland stations fulfilled the $40^{\circ}$ magnetic meridian chain. This enables the study of interhemispheric magnetic asymmetries (Kim et al. 2013; Hartinger et al. 2017; $\mathrm{Xu}$ et al. 2020).

The AAL-PIP system is designed to operate remotely and autonomously in a configurable manner for at least 3-5 years of mission duration. For the details of system operation, see (Clauer et al. 2014; Xu et al. 2019). Six AAL-PIP systems, including four CASES receivers at PG2, PG3, PG4, and PG5, are presently deployed. The system locations are shown as red hollow stars in Fig. 1.

The operation of the CASES receivers must follow a number of protocols in order to accommodate the system engineering requirements of AAL-PIP. The considerations include limited power arrangement (due to the unique remote locations), AAL-PIP's thermal control protocols, data storage capacity, and Iridium communication system bandwidth. The CASES GPS receiver operation is highly autonomous (based on the computer script from operation manager) and re-configurable (by conducting remote firmware update via the Iridium communication network).

There are a number of CASES data collection strategies that are tailored to fit different scientific objectives. Low rate data can be collected when scintillation measurements are not needed. This strategy has the advantage

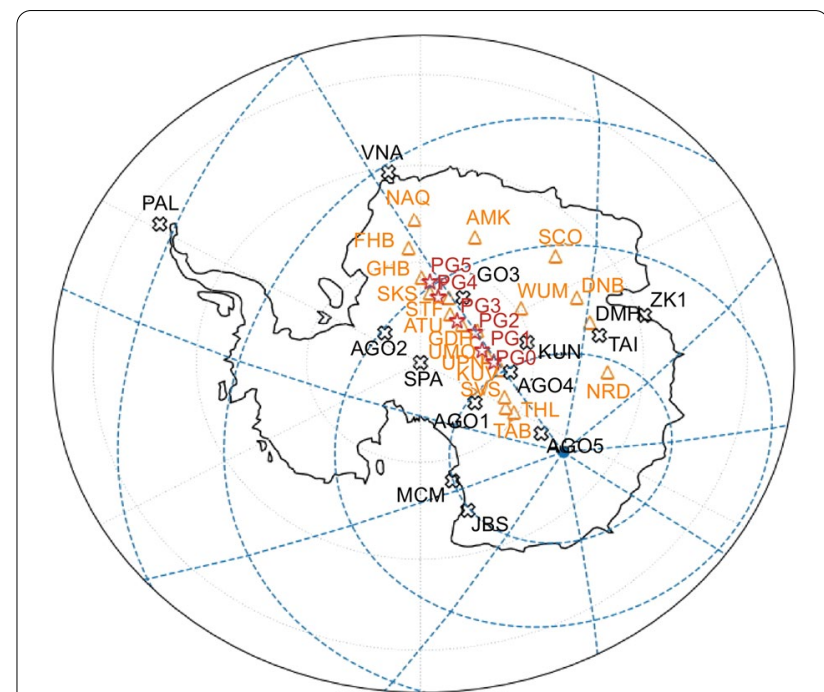

Fig. 1 Antarctic $40^{\circ}$ magnetic meridian chain (AAL-PIP stations are shown in red)

of generating smaller datasets that can be more readily transferred over the Iridium. Higher rate data can be collected in a variety of modes where the time and duration of data collection are altered to examine substorm effects on the ionosphere (e.g., data from a few hours near local midnight are collected), dayside transients (e.g., a few hours near local noon), or conduct a more variable sampling of all magnetic local time (e.g., turn on for one hour, off for three hours) (Kim et al. 2014). CASES can also be run continuously, but this runs the risk of overheating the system. Regardless of the mode of data collection, CASES data files tend to be the largest collected by AAL-PIP, and there is often a backlog of data files awaiting transfer. Standard practice in the recent seasons has been to transfer all CASES measurements from the previous season when the system enters low power mode in April. Low rate data is typically collected around local dawn, noon, and dusk, while high rate data is collected in a period around local midnight. A GPS data inventory is available on this MIST website: "http://mist.nianet.org/GPS/".

\section{GPS data processing}

In AAL-PIP, the CASES receivers are utilized to generate several different data products, including Receiver Independent EXchange format (RINEX) observation files, CASES log files, TEC estimations, ULF-wave modulation stack plots, and ice sheet movement measurements. This section briefly overviews the data processing methods for each data product. A GPS data processing routine flowchart is given in Fig. 2.

First, the binary raw GPS data transferred from the AAL-PIP system are converted into human-readable 


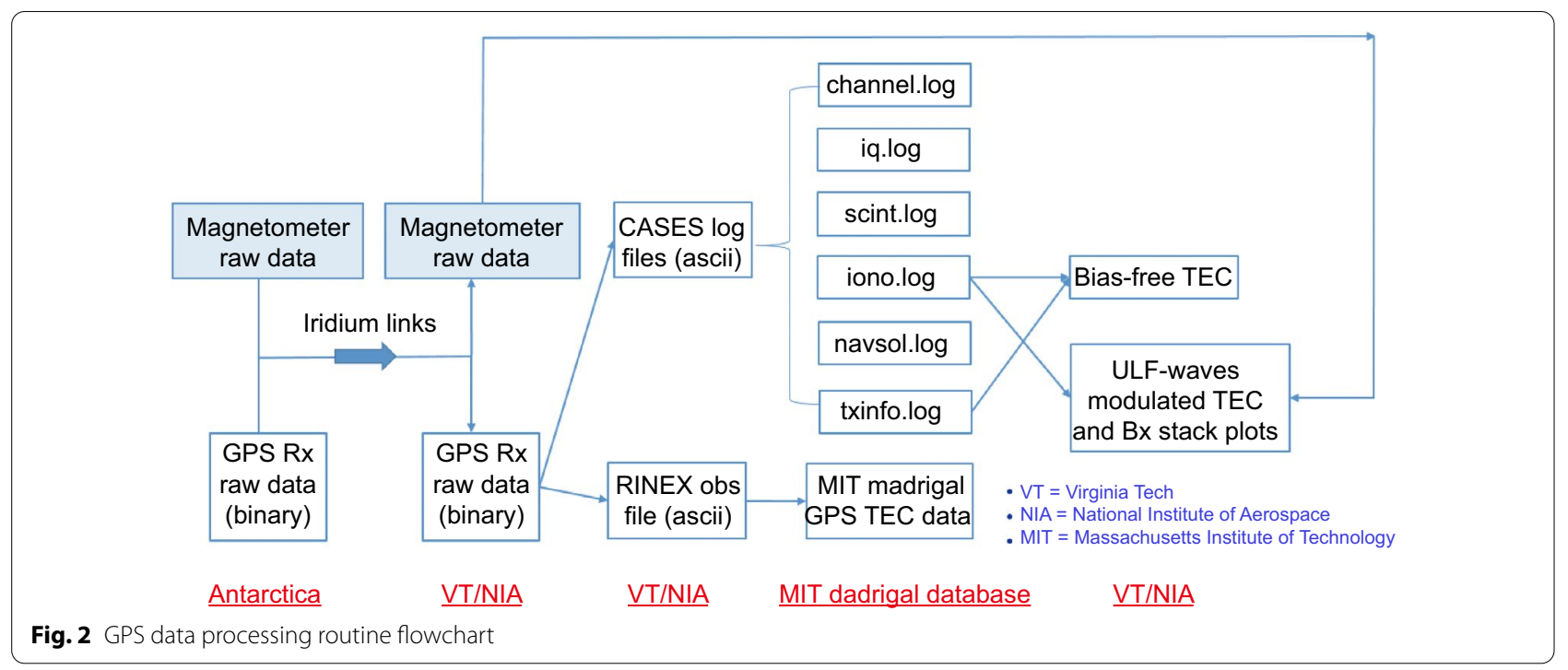

format data. By default, CASES raw receiver data are automatically collected and saved as compressed binary files (.dat) with a sampling cadence of $10 \mathrm{~min}$. As the receivers are not continuously operated, the availability of raw binary data depends on the operation modes. The daily aggregated ".dat" files are converted to "bin" files by using "sbcclient" executable program (a CASES client-side software available from ASTRA, http://cases. astraspace.net/software.html). Then RINEX files and CASES log files are extracted from ".bin" files. For Antarctic AAL-PIP CASES data processing, only observation files are extracted (in RINEX version 2.11 ASCII format) as the GNSS navigation files can be downloaded from the NASA Crustal Dynamics Data Information System (CDDIS) (NASA 2010). The CASES log files, in ASCII format, consist of low rate data in "channel.log", high rate data in "iq.log", scintillation data in "scint.log", ionospheric (raw TEC) data in "iono.log", navigation data in "navsol.log", and transmitter data in "txinfo.log". Formats of these log files can be found at ASTRA, http://cases. astraspace.net/documentation/.

The RINEX (v2.11) observation files (collected from the AAL-PIP's PG2, PG3, PG4, and PG5 stations) are used by the MIT Madrigal GPS database MIT to generate global GNSS/GPS TEC maps (Vierinen et al. 2016). GNSS TEC is the total number of electrons integrated along the lineof-sight between a GNSS satellite and a GNSS receiver antenna. Unless the GNSS satellite's elevation angle is $90^{\circ}$, the actual integrated value is referred as slant TEC. The "iono.log" files can directly provide the "pseudorange slant TEC" and "raw carrier-phase slant TEC". Using a first order residual fitting method, the "fitted relative slant TEC" can be obtained by smoothing the "pseudorange

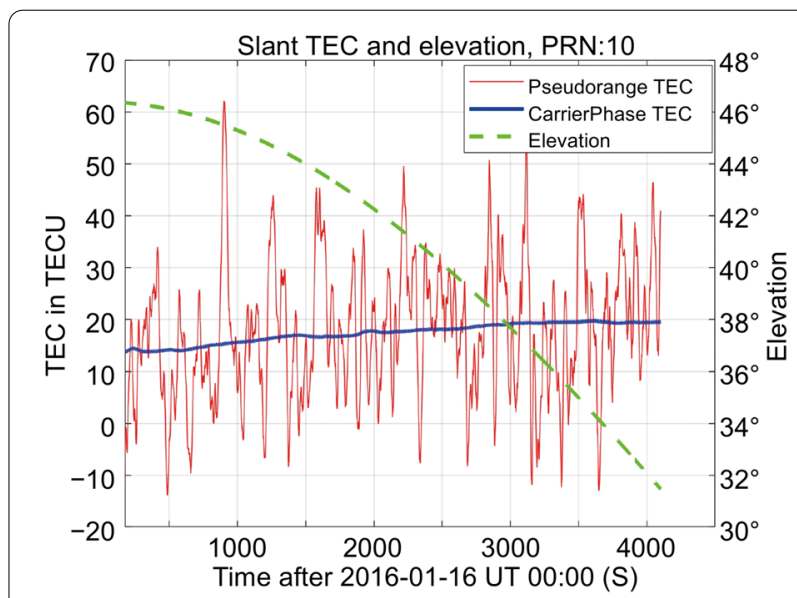

Fig. 3 Smoothing pseudorange TEC using carrier-phase TEC. The blue line is the "fitted relative slant STEC"

slant TEC" by "raw carrier-phase slant TEC". A sample case is shown in Fig. 3 for illustration, where the blue line is the "fitted relative slant STEC" and TECU is Total Electron Content Units with 1 TECU being $1 \times 10^{16}$ electrons per $\mathrm{m}^{2}$. The term "relative" here means the slant TEC values contain Differential Code Biases (DCB). A DCB estimation and elimination process (based on a differential linear least-squares method developed by Gaposchkin and Coster (1993) and implemented by Peng et al. (2020) is taken to further process the "fitted relative slant TEC" into "bias-free absolute slant TEC". The negative STEC/ TEC values are avoided by using the "Zero TEC" method described in Rideout and Coster (2006). A sample case to correct the TEC DCB is given for illustration in Fig. 4. 

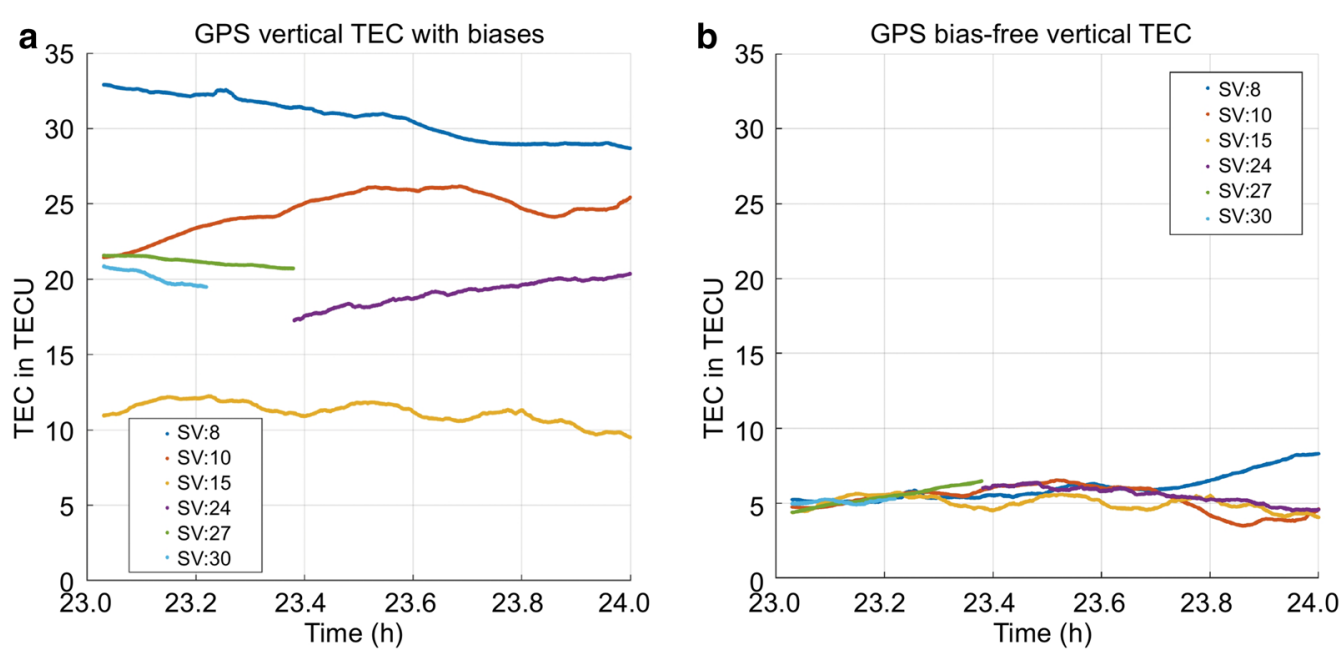

Fig. 4 GPS vertical TEC plots a DCB included b DCB eliminated

This dataset was collected by PG3's CASES receiver on January 13, 2016. Before and after the DCB estimation and correction, the Vertical TEC (VTEC) plotted for different GPS Space Vehicles (SV) are shown in Fig. 4a, b, respectively. Starting from $23.7 \mathrm{~h}$, the divergence of GPS bias-free vertical TECs between different Pseudorandom Noise (PRN) is possibly associated with relatively low PRN elevation angle (e.g., elevation angle of SV \#8< 3016) and ionospheric irregularities (e.g., electron density structures). To calculate VTEC, the elevation angles of a GNSS satellite calculated from ephemeris are used to compute a thin shell mapping function $(F)$ (Klobuchar 1987): $F=1+16 \times(0.53-E)^{3}$, where $E$ is the elevation angle in semicircles.

A software tool was developed by Scales et al. (2019) to produce carrier-phase TEC stack plots with ULF wave modulation visibility. Pc 5 and Pc 4 waves with frequencies of 1 to $22 \mathrm{mHz}$ were mainly investigated [definitions of Pc waves can be found in Jacobs et al. (1964)]. The overall functionality of the tool is to autonomously input GPS TEC and magnetometer data then produce the plots which superimpose the fluctuations of TEC and geomagnetic field respectively based on a frequency band of interest. As a sample case using two GPS PRNs (5 and 9), GPS TEC and magnetic field superposition stack plots from four AAL-PIP systems are shown in Fig. 5. In each panel, carrier-phase TEC after detrending and high-pass filtering is plotted in blue with the values respect to the left $y$-axis in TECU, while geomagnetic field magnitude $(\mathrm{Bx})$ after the same detrending and high-pass (frequency) filtering is plotted in orange with the values respect to the right $y$-axis in nT. After 25 mins, the wave modulation on Bx can be clearly seen and wave activity with a similar period of roughly 4-5 mins is also seen in TEC at most
locations/PRNs. A light-correlation pattern between TEC and Bx can arguably be identified. Note there are multiple mechanisms that could lead to a TEC ULF wave modulation, some of which may have TEC modulations in phase with $\mathrm{Bx}$ and some out of phase.

\section{Space weather observations}

Solar storms can induce ionospheric irregularities in the polar region (e.g., auroral precipitation) and cause strong GPS scintillations. The previous work using AAL- PIP CASES receivers has shown that, the Pi2 and Pi1B types of ULF waves are correlated with GPS scintillation in the high-latitude ionosphere possibly due to a formation of plasma instability (Kim et al. 2014). A novel three-dimensional electromagnetic wave propagation model called "Satellite-beacon Ionospheric-scintillation Global Model of the upper Atmosphere (SIGMA)" is developed to simulate GNSS scintillations on the ground in the highlatitude regions (Deshpande et al. 2014). Two scintillation cases on 9 January 2014 were simulated using SIGMA with the help of available auxiliary data and the number densities and spectral indices were derived for PG2 and PG3. The results indicate that the two scintillation cases have the same source which may be related to the substorm during the same period (Deshpande 2014).

ULF waves (also known as geomagnetic pulsations) are a class of electromagnetic waves with frequencies from 1 $\mathrm{mHz}$ to $1 \mathrm{~Hz}$ (Jacobs et al. 1964). ULF waves were first observed via ground-based measurements of the 1859 Great Aurora events (Stewart 1861). ULF waves have the potential to modulate TEC and these modulations can potentially affect GPS signal traffic through our atmosphere (Pilipenko et al. 2014), but the mechanism(s) causing the modulation are not well understood. Utilizing 


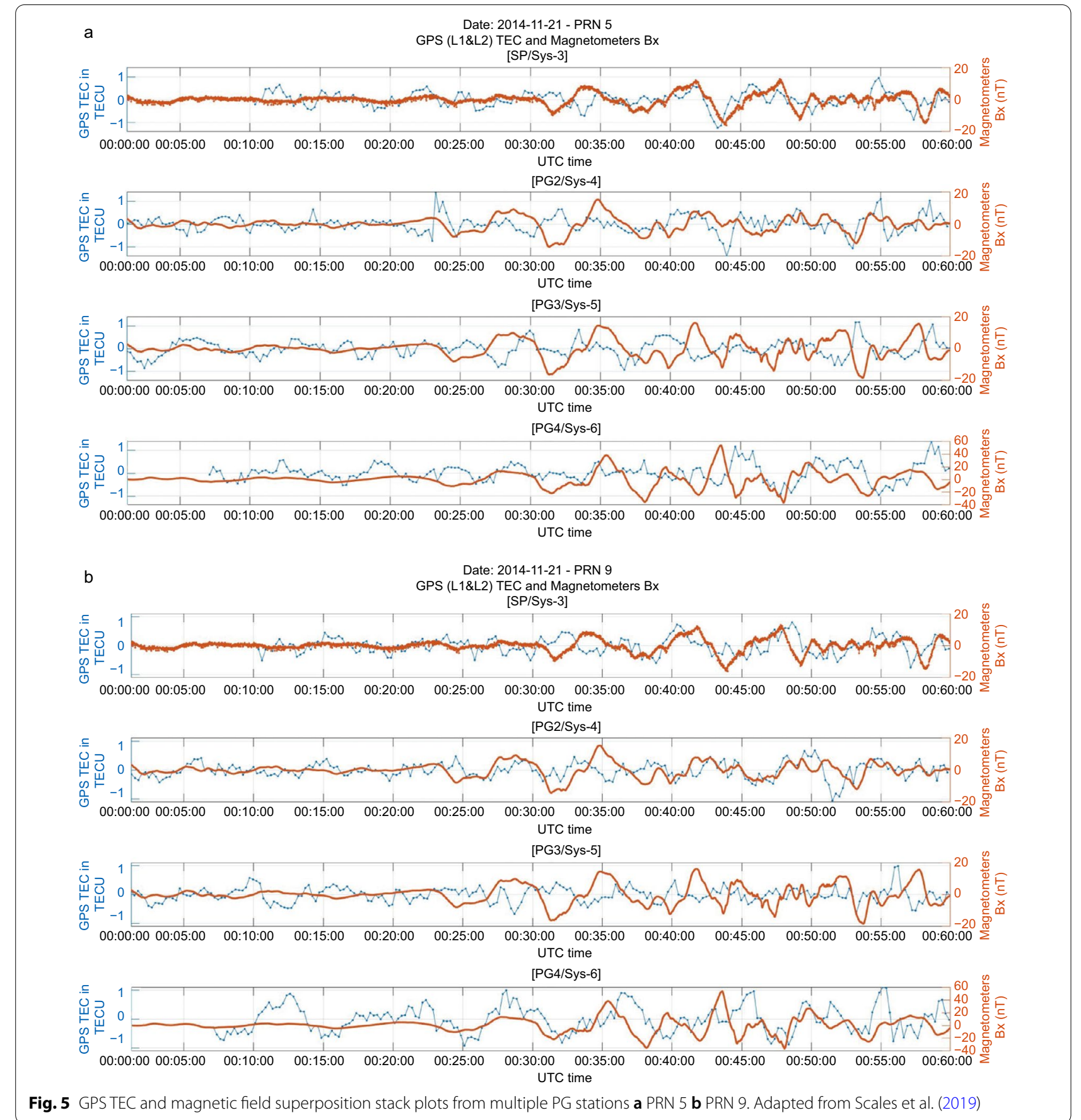

AAL-PIP, ULF waves were observed over Antarctica using multi-station GPS TEC and magnetometer (Scales et al. 2019; Xu et al. 2019). As shown in Fig. 6, a ULFwave modulated TEC event observed by AAL-PIP was reported by $\mathrm{Xu}$ et al. (2019). In this case, we can clearly see the synchronized fluctuations between the blue waveforms (TEC data) and the orange waveforms (magnetic field $\mathrm{Bx}$ ). By analyzing a series of these plots that span all the available satellites and monitoring stations for a given time period, researchers are able to characterize the spatial and temporal variations in TEC and determine whether these are related to magnetospheric wave activity (produces magnetic signature) or smaller scale structures in the ionosphere (e.g., ionospheric irregularities with weak/no magnetic signature). Additionally, since AAL-PIP stations are spread across the same magnetic 


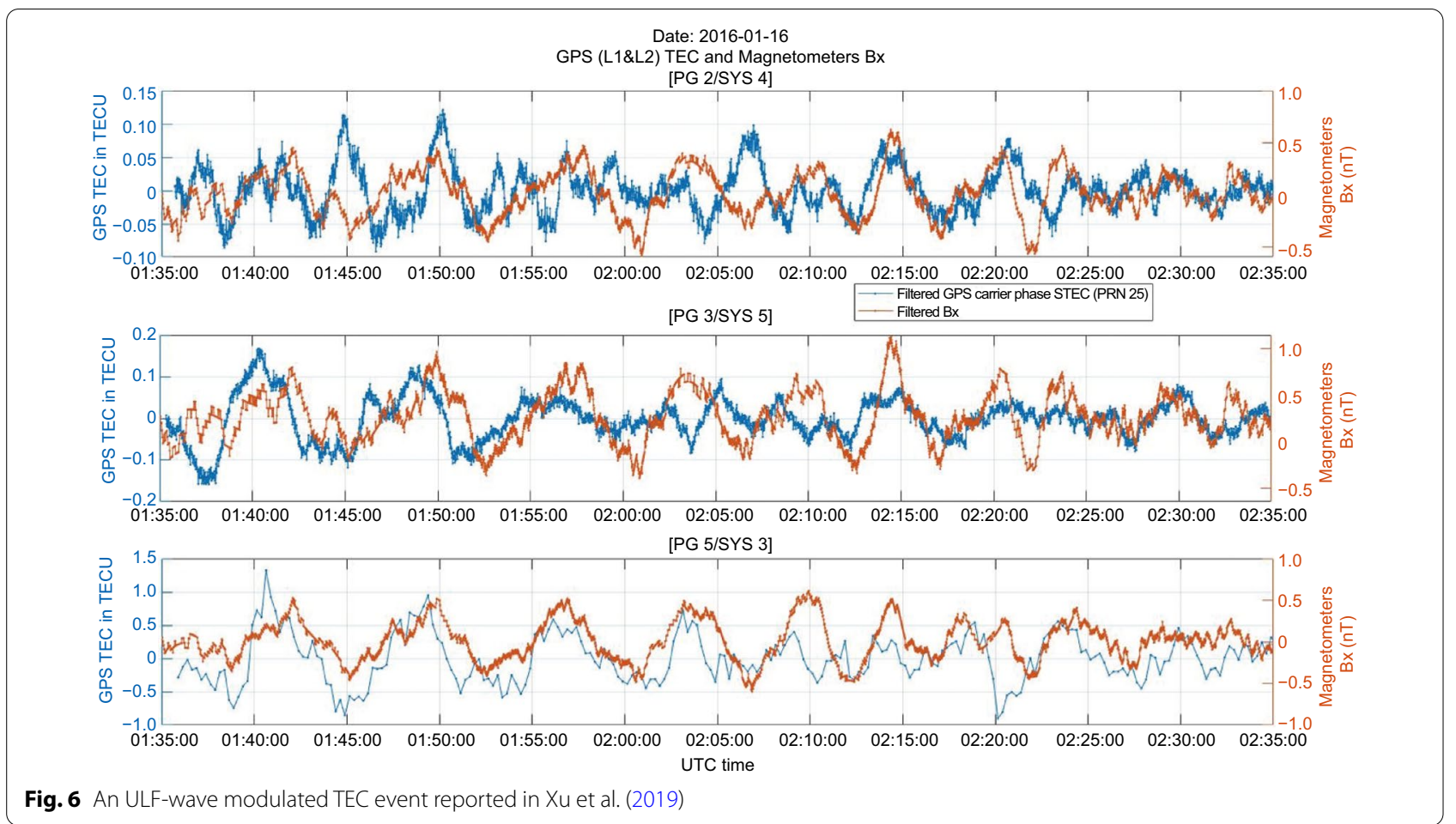

meridian, it is possible at times to infer wave propagation speeds. Beside Antarctica (southern hemisphere highlatitude region), similar ULF-modulated TEC events were detected in the northern hemisphere high-latitude region as well (Pilipenko et al. 2014; Watson et al. 2015).

The network of AAL-PIP ground-based GPS receivers can potentially team up space-based GNSS receivers on Low Earth Orbiting (LEO) satellites for conjunctive geospace measurements of ULF waves. Further details will be discussed in Section A new space-based observation technique.

\section{Active measurement of high-latitude ionospheric structure and impacts on GNSS}

Modification of the ionosphere by high power HF radio waves has had a long and rich history dating back to the 1960's (e.g., Gurevich 2007). These experiments consider the creation of phenomenon in the ionosphere due to the interaction of the high-power transmitted wave (called the pump or pumping wave) with the ionosphere. Typically, the pump frequency $\omega_{0}$ is in the range from 2 to 8 $\mathrm{MHz}$ and maximum transmitter power in the range of several megawatt $(\mathrm{MW})$. The interaction altitude with the ionosphere is typically in the range of $200 \mathrm{~km}$. This type of active space experiment has the advantages over typical passive experiments that ionospheric phenomenon can arguably be studied in more careful detail since initial conditions can be prescribed and be compared to theory and models more readily. A plethora of physical processes due to nonlinear plasma physics are produced during this type of experiment which include the creation of artificial aurora (i.e. optical emissions), artificial ionization layers, heating and acceleration of electrons, production of secondary radiation (called Stimulated Electromagnetic Emissions or SEE), and others. Because of the increase in electron temperature during these experiments due to electron collisions under motion in the pump wave, these experiments are often called "ionospheric heating experiments".

An important phenomenon, relevant to GNSS scintillations, associated with ionospheric heating experiments is the production of artificial ionospheric irregularities on spatial scales from kilometers down to centimeters (Gurevich 2007). The irregularities, often studied, are of spatial scale of several kilometers along the magnetic field and 10 meters (decameter) across the magnetic field and are therefore called Artificial Field Aligned Irregularities (AFAI) and produced near the altitude when the pump frequency $\omega_{0}$ is near the local upper hybrid frequency $\omega_{u h}$. It has been known that there are particularly strong effects on ionospheric heating related phenomenon when $\omega_{0}$ is near a harmonic of the electron gyrofrequency $n \Omega_{c e}$ (where $n$ is harmonic number and $\Omega_{c e} \approx 1.4$ $\mathrm{MHz}$ ) and also $\omega_{u h}$, a condition called double resonance. These so-called "gryoharmonic effects" are also prevalent in other phenomenon created during ionospheric 
heating experiments. There is potential to produce GNSS amplitude and phase scintillation by the decameter spatial scales across the magnetic field. These lie within the Fresnel scale possibly to produce scintillations in the classical manner. It should be noted that inside these decameter scale irregularities are embedded smaller scale irregularities $(10 \mathrm{~cm}$-scale $)$ that may be produced by the plethora of nonlinear processes during the heating experiment that are expected to be relevant to GNSS scintillations and have only recently been considered.

It was first proposed by Gurevich and Zybin (2006) that strong irregularities termed Super Small Striations (SSS) existing on $10 \mathrm{~cm}$ scales across the magnetic field could produce GNSS scintillation and also the scintillation of other Ultra-High Frequency (UHF) signals. These SSS irregularities could be generated by nonlinear processes that produce plasma waves in this scale size range during heating. Particularly, perpendicularly (to the magnetic field) propagating Electron Bernstein (EB) waves which are produced during heating associated parametric decay processes are of $10 \mathrm{~cm}$ spatial scale sizes and would be embedded with AFAI. The EB waves have long been thought to play a crucial role in producing SEE that are commonly observed during ionospheric heating experiments (Leyser 2001). Therefore, the observation of both specific SEE spectral lines and the enhancement of GNSS TEC fluctuations could provide evidence of SSS producing GNSS scintillations.

Milikh et al. (2008) reported the first observations of GNSS scintillations produced by SSS that could be modulated by turning the heating 'on' and 'off' at the Highfrequency Active Auroral Research Program (HAARP) facility near Gakone, Alaska. The scintillations were typically observed about $10 \mathrm{~s}$ after heater turn-on. Milikh et al. (2008), Najmi et al. $(2014,2015)$ also noted the correlation of the GNSS scintillation with specific SEE spectral lines which are measured with ground-based HF receivers near the HAARP transmitter. There was strong correlation with the so-called Broad Upshifted Maximum (BUM) SEE spectral line and weaker correlation with the so-called Downshifted Maximum (DM) SEE spectral line. The DM is shifted below the pump frequency in the SEE spectrum by approximately $10 \mathrm{kHz}$ and has long been associated with the generation of Field-AlignedIrregularities (FAIs) of spatial scales perpendicular to the magnetic field of order 10 meters (Leyser 2001). The BUM, whose maximum amplitude is upshifted (rather than downshifted) from the pump frequency by tens of $\mathrm{kHz}$, with similar bandwidth, has been proposed to be associated with EB waves of spatial scale of order 10 $\mathrm{cm}$ and propagation perpendicular to the magnetic field embedded in the 10 meter irregularities. Also, the BUM exists for $\omega_{0}$ in close proximity to $n \Omega_{c e}$ as was the case for previous experiments where $\omega_{0} \approx 3 \Omega_{c e} \approx 4.2 \mathrm{MHz}$. The work of Mahmoudian et al. (2018) attempted to provide more detailed comparisons of the SEE spectral line temporal evolution, namely the BUM, (indicating existence of the SSS) and GNSS scintillations when cycling the HAARP ionospheric heating cycle on and off for $\omega_{0} \approx 4 \Omega_{c e} \approx 5.7 \mathrm{MHz}$.

The relationship between the GPS phase scintillations and SSS irregularities was proposed by Najmi et al. (2014). The measured GPS phase fluctuation $\Delta \phi$ upon passing through the heated volume due to the SSS irregularities can be written as follows

$$
\Delta \phi=\frac{\omega}{c} \times \frac{\Omega^{2} c e}{\omega^{2}} \times \frac{\delta n_{e}}{n_{e}} l
$$

where $\omega$ is the GPS radian frequency (L1 or L2), $c$ is the speed of light, $\delta n_{e} / n_{e}$ is the electron density fluctuation amplitude of the SSS irregularities, and $l$ is the propagation length of the GNSS signal through the irregularities. The SSS irregularity density fluctuation can be related to the slant TEC fluctuation $(\triangle \mathrm{STEC})$ by

$$
\frac{\delta n_{e}}{n_{e}}=4.37 \times \frac{\Delta \mathrm{STEC}}{l}
$$

Therefore, the measured differential phase fluctuations between the GPS L1 $\left(\omega_{1}\right)$ and L2 $\left(\omega_{2}\right) \Delta \phi_{12}$ can be used to determine $\triangle$ STEC which is a proxy for the GPS phase scintillations due to the SSS, namely

$$
\triangle \mathrm{STEC}=\frac{0.75 \times \omega_{1} \Delta \phi_{12}}{\left(\omega_{1} / \omega_{2}\right)^{2}-1}
$$

Figure 7a shows the STEC phase fluctuation $\Delta$ (STEC) temporal evolution for an averaging over 10 heating cycles reported by Mahmoudian et al. (2018). The experiments were performed in 2014 at the HAARP facility. It can be seen that $\triangle$ (STEC) increases to a maximum within about $1 \mathrm{~s}$ of the beginning of the heating cycle and after the saturation amplitude is achieved, begins to slowly reduce to its preheating value. This indicates the increase of GPS scintillation during the heating cycle and also the ability to modulate the phase scintillations with the ionospheric heater. The signal from GPS PRN 25 was used with the heater transmitter pointed in the PRN direction with a readjustment of elevation and azimuth every $5 \mathrm{~min}$ to track the GPS satellite appropriately. The heating cycle lasted for $100 \mathrm{~s}$ 'on' with an 'off' period of $20 \mathrm{~s}$ as can be seen in Fig. 7. During the 'on' period of each cycle a new frequency stepped by $30 \mathrm{kHz}$ near $\omega_{0} \approx 4 \Omega_{c e} \approx 5.7 \mathrm{kHz}$ was used to enhance the production of SSS by approaching the gyroharmonic. The frequencies used were in the range from 5.67 to $5.94 \mathrm{MHz}$. The experiments performed at HAARP in 2013, with a different heating cycle 

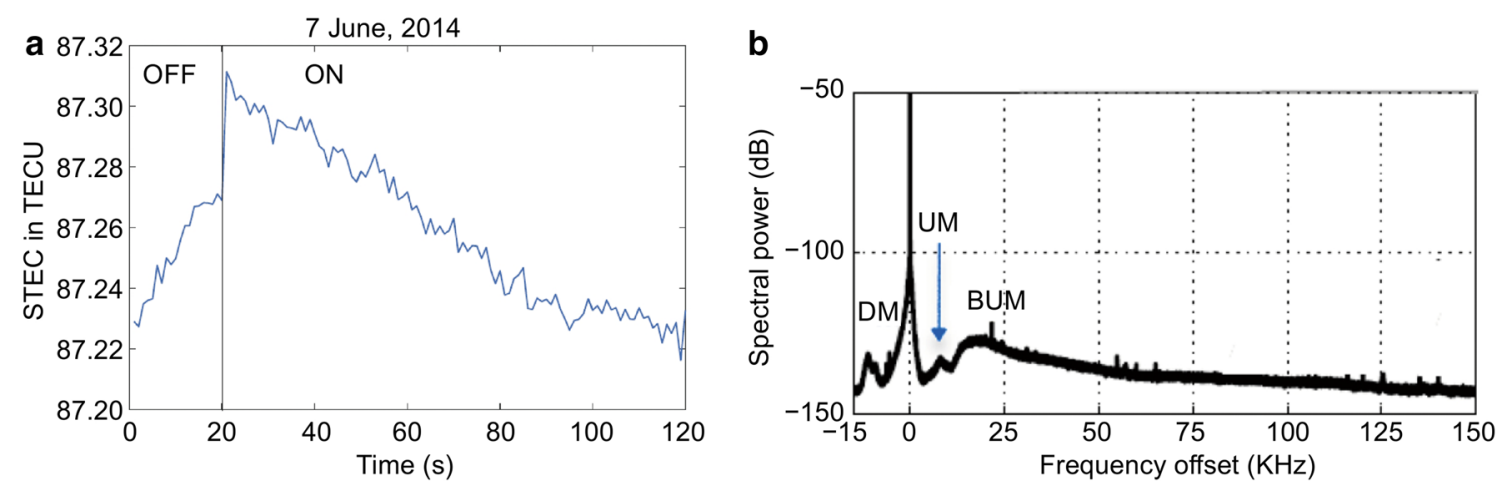

Fig. 7 a Increase in STEC fluctuation $(\Delta(S T E C))$ at the beginning of a 100 s ionospheric heating experiment cycle which are proposed as a proxy for GPS phase scintillations. Subsequent decay is proposed to be due to nonlinear processes involving EB waves of spatial scale $10 \mathrm{~cm}$ which produce the scintillations. $\mathbf{b}$ Spectrum of Stimulated Electromagnetic Emissions (SEE) observed from ground-based receivers during the heating experiment for $\omega_{0}=5.67 \mathrm{kHz}$. The spectral line offset $10 \mathrm{kHz}$ below the pump frequency (DM) is associated with irregularity spatial scales of $10 \mathrm{~m}$ across the magnetic field and proposed to be associated with irregularities nearer the Fresnel scale resulting in only weak scintillations. The spectral line upshifted by $20 \mathrm{kHz}$ (BUM) is proposed to be the proxy for $10 \mathrm{~cm}$ so-called Super-Small-Striations (SSS) that produce the strong GPS phase scintillations observed during the experiment. (Adapted from Mahmoudian et al. (2018))

and satellite PRN 07, show the similar temporal evolution behavior of a relative rapid increase at the beginning of the cycle with saturation after roughly 1 s and then slow decay to the preheated value.

Also correlated with the observations of stronger GPS scintillations during the heating was SEE spectral lines showing the appearance of the SEE BUM spectral line proposed as a proxy for EB waves of spatial scale of order $10 \mathrm{~cm}$ that are expected to provide GPS signal scattering resulting in GPS scintillations. Figure $7 \mathrm{~b}$ shows an average over the 10 heating cycles of the SEE spectrum from $15 \mathrm{kHz}$ below to $150 \mathrm{kHz}$ above $\omega_{0}$. The strongest BUM observed is for the pump frequency of $5.67 \mathrm{MHz}$. The DM SEE spectral line is roughly at a $10 \mathrm{kHz}$ offset below the pump frequency (i.e. 0 offset is $\omega_{0}$ ). This spectral line is often considered as a signature of AFAI with spatial scale of $10 \mathrm{~m}$ across the magnetic field. The BUM, however, is proposed as a signature of $10 \mathrm{~cm}$ spatial scale SSS embedded within the $10 \mathrm{~m}$ irregularities. In Fig. 7a it is the spectral line with maximum upshifted offset near $20 \mathrm{kHz}$ and similar bandwidth. Mahmoudian et al. (2018) considered the nonlinear plasma simulation model of $\mathrm{Xi}$ and Scales (2001) to interpret the decay of the GPS scintillations after saturation (Fig. 7a) to the damping of the EB waves from cyclotron damping (Tripathi and Liu 1993). This evolution is observed in the nonlinear plasma simulations of the 4-wave parametric decay instability that ultimately produces the EB waves from the original pump wave (Huang and Kuo 1994) as shown in Fig. 8. Figure 8a shows the growth rate of the production of SSS irregularities by the interaction of the high power HF radio wave with the ionosphere. These irregularities are in the decimeter (i.e. $10 \mathrm{~cm}$ ) range and of the order of the

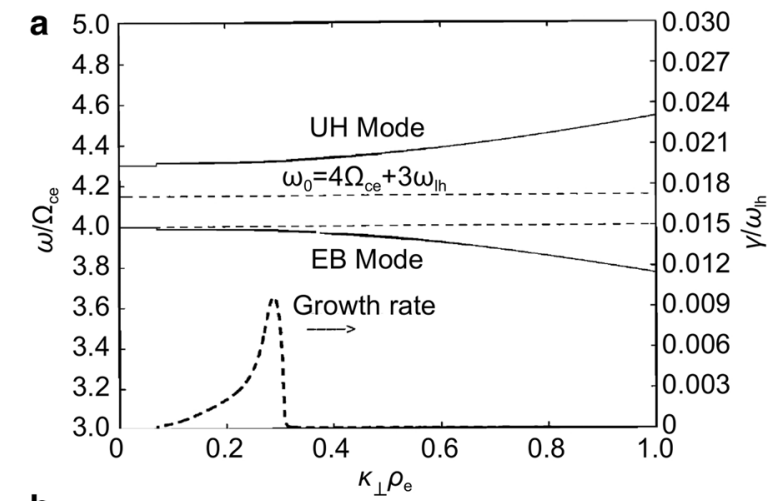

b

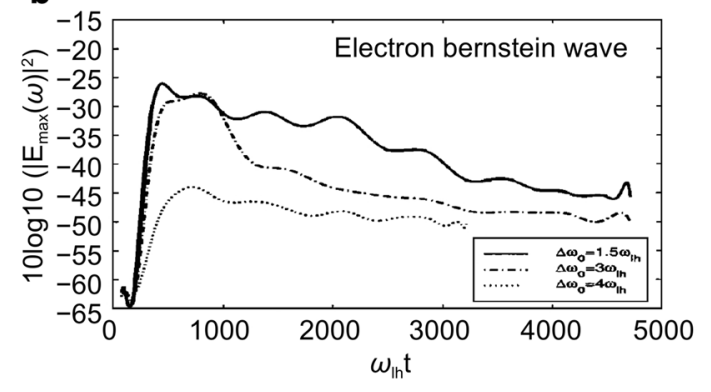

Fig. 8 a Dispersion relation (i.e. frequency, $\Omega$, versus perpendicular wavenumber, $k_{\perp}$ and growth rate $(\Upsilon)$ of Strong Super Small Striation (SSS) irregularities generated during an ionospheric heating experiment), proposed to produce GNSS phase scintillations. Note Electron Bernstein (EB) waves are generated in the range $k_{\perp} \rho_{e} \sim 0.3$, which implies decimeter SSS irregularities. b Nonlinear numerical simulation of SSS showing temporal damping of EB SSS which aligns with the reduction in GPS phase scintillations in Fig. 7 (Mahmoudian et al. 2018) (Adapted from Xi and Scales (2001)) 
GNSS transmit wavelength. Figure 8b shows the damping of the EB waves due to nonlinear plasma evolution through the heating of plasma electrons. Although, more careful modeling work in line with $\mathrm{Xi}$ and Scales (2001) is necessary, the correlation of the strong GPS phase scintillations with SEE spectral lines associated with $10 \mathrm{~cm}$ SSS irregularities (namely the BUM) indicate that SSS is indeed important for producing GNSS scintillations during ionospheric heating experiments.

\section{Modelling and observation of mid-latitude ionospheric irregularity effects on GNSS}

Although mid-latitude ionospheric irregularities are less well understood than high and low latitude ionospheric irregularities (Kelley 2009), they are important to the production of GPS scintillations (Kintner et al. 2007). Over the past decade, the HF SuperDARN space weather radar network (Greenwald et al. 1995) has been extended to mid-latitudes, i.e., the sub-auroral region, and important new understanding of characteristics of mid-latitude irregularities were made (Greenwald et al. 2006) which may have useful implications for GNSS scintillation production. An important conclusion of the mid-latitude SuperDARN radar measurements is that even during quiet times of geomagnetic activity there is nearly continual mid-latitude ionospheric irregularities whose source is currently not well understood (Ribeiro et al. 2012). The resolution of this forefront problem may have important implications for understanding GNSS scintillations at mid-latitudes.

Typically, irregularities of spatial scales of hundreds of meters are associated with GNSS scintillations, due to the irregularity spatial scales relative to the Fresnel scale. However, the SuperDARN HF radar returns are associated with shorter irregularity spatial scales of tens of meters (decameter) which produce coherent radar echoes through Bragg scattering. Joint experiments performed by the MIT Haystack Observatory and the Virginia Tech SuperDARN HF space weather radar group indicated the opposed ionospheric electron temperature and density gradients in the regions of radar scatter (Greenwald et al. 2006) associated with mid-latitude ionospheric irregularities. Such conditions of opposing density and temperature gradients have been associated with the Temperature Gradient Instability (TGI) (e.g., Hudson and Kelley 1976) which has also been proposed as an important irregularity generation mechanism for mid-latitude GPS scintillations (Kintner et al. 2007). Subsequent work considered the possibilities of the TGI being able to produce irregularities in both the $100 \mathrm{~m}$ and $10 \mathrm{~m}$ spatial scales simultaneously which would produce both GNSS scintillation and SuperDARN radar scatter (de Larquier et al. 2014; Eltrass et al. 2014, 2016). Although it could be shown from local linear plasma instability theory that the TGI could directly produce irregularities in the 100 $\mathrm{m}$ scales size relevant to GNSS scintillations and also 10 $\mathrm{m}$ scales relevant to SuperDARN radar scatter separately (Eltrass et al. 2014), there was still the important question of the possibility of the TGI producing both larger $100 \mathrm{~m}$ and smaller $10 \mathrm{~m}$ spatial scales simultaneously. If this was the case, then perhaps mid-latitude SuperDARN radar observations could provide an insight into the occurrence of GNSS scintillations at mid-latitudes.

The possibility of the investigation of this premise could not be considered with the TGI linear plasma instability theory and required the utilization of more advanced plasma simulations to consider the nonlinear evolution of the irregularity development produced by the TGI from larger to smaller spatial scales through turbulent cascading processes. Eltrass and Scales (2014) developed a plasma simulation model using gyro-kinetic and Monte-Carlo plasma simulation techniques to consider nonlinear cascading of TGI irregularities from 100 $\mathrm{m}$ to $10 \mathrm{~m}$ spatial scales which would produce simultaneous impact of irregularities at both GNSS scintillation spatial scales and SuperDARN radar scatter from shorter scale irregularities.

Figure 9 shows the configuration of the Two-Dimensional (2D) gyro-kinetic plasma simulation model with Monte Carlo electron-neutral collisions utilized by Eltrass and Scales (2014) to study the nonlinear evolution of the TGI. The opposing temperature and density gradients $(\mathrm{Kn}, \mathrm{KT})$ produce diamagnetic drifts ultimately responsible for the development of the TGI turbulence which can be classified as a collisional drift wave (Mikhailovskii 1974). These opposed gradients have been estimated using MIT Haystack Observatory Incoherent Scatter Radar (ISR) observational measurements by de Larquier et al. (2014). The TGI turbulence propagates in the $2 \mathrm{D} y-z$ simulation plane, nearly perpendicular to the geomagnetic field $\mathrm{B}$ ( $z$ direction) in the $y$ direction. In the simulations, the turbulence was seeded at $1 \mathrm{~km}$ spatial scale which is somewhat larger than scale sizes to produce GNSS scintillations. Figure 9a shows the wavenumber spectrum of ionospheric plasma density structures after the saturation of the TGI turbulence. The black curves is the early time spectrum $\left(\Omega_{c i} t=100\right.$ where $\Omega_{c i}$ is the ion cyclotron frequency) which shows the power at the seed turbulence near $1 \mathrm{~km}$ spatial scale. After the nonlinear saturation of the turbulence on later time scales $\left(\Omega_{c i} t=1000\right)$, it can be seen that a broad spatial spectrum from the 1 $\mathrm{km}$ seed wavenumber spectrum down to $10 \mathrm{~m}$ spatial scale in the red curve. This indicates that TGI turbulence produced at relatively longer spatial scales may go through nonlinear turbulent cascading, and produce 


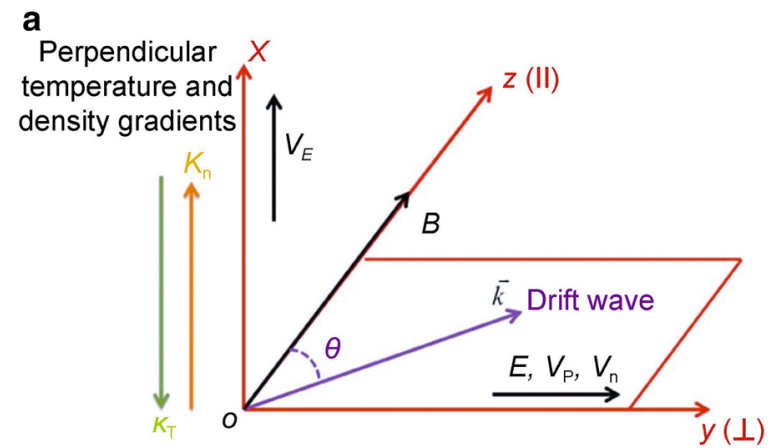

b

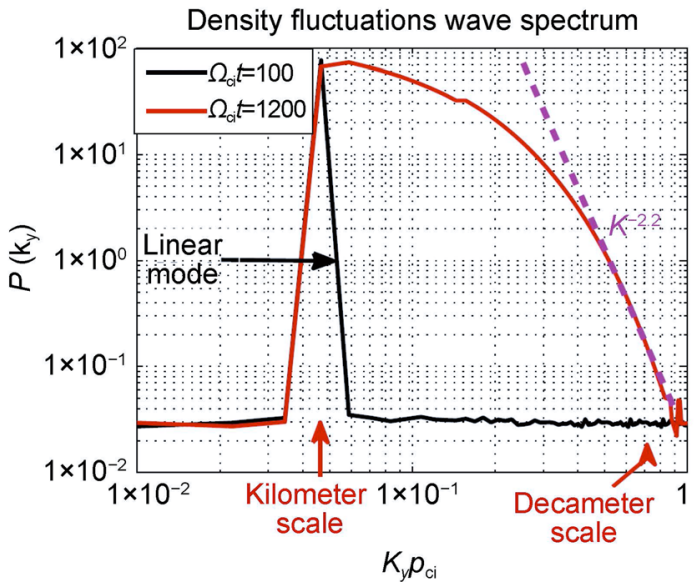

Fig. 9 a Configuration of a 2D plasma simulation used to study development of irregularities in the mid-latitude ionosphere. Primary generation is the Temperature Gradient Instability (TGI) due to opposed density and temperature gradients in the sub-auroral region. $\mathbf{b}$ Irregularity spatial spectrum in the y direction (propagation direction) from the simulation showing development of irregularities from cascading turbulence from the $\mathrm{km}$ spatial range down to decameter spatial scales that impact both GNSS scintillations and also HF space weather radar scattering (Adapted from Eltrass and Scales (2014))

irregularity scales both in the $100 \mathrm{~m}$ range, which produces GNSS scintillations, as well as $10 \mathrm{~m}$ spatial scales which produces SuperDARN radar coherent scatter. Therefore, SuperDARN radar scatter at mid-latitudes can provide an important insight on GNSS scintillations (and vice versa). The irregularity spectrum shown on Fig. 9b also indicates a saturated turbulent spectral index of approximately $k^{-2.2}$ (where $\mathrm{k}$ is the wavenumber) for the ionospheric irregularities produced by the TGI.

A more careful appraisal of the TGI as a possible mechanism for GNSS scintillations was made by a comparison of the simulation spectral characteristics (i.e., wavenumber power spectral index) with mid-latitude GNSS scintillation data as well as in situ measurements
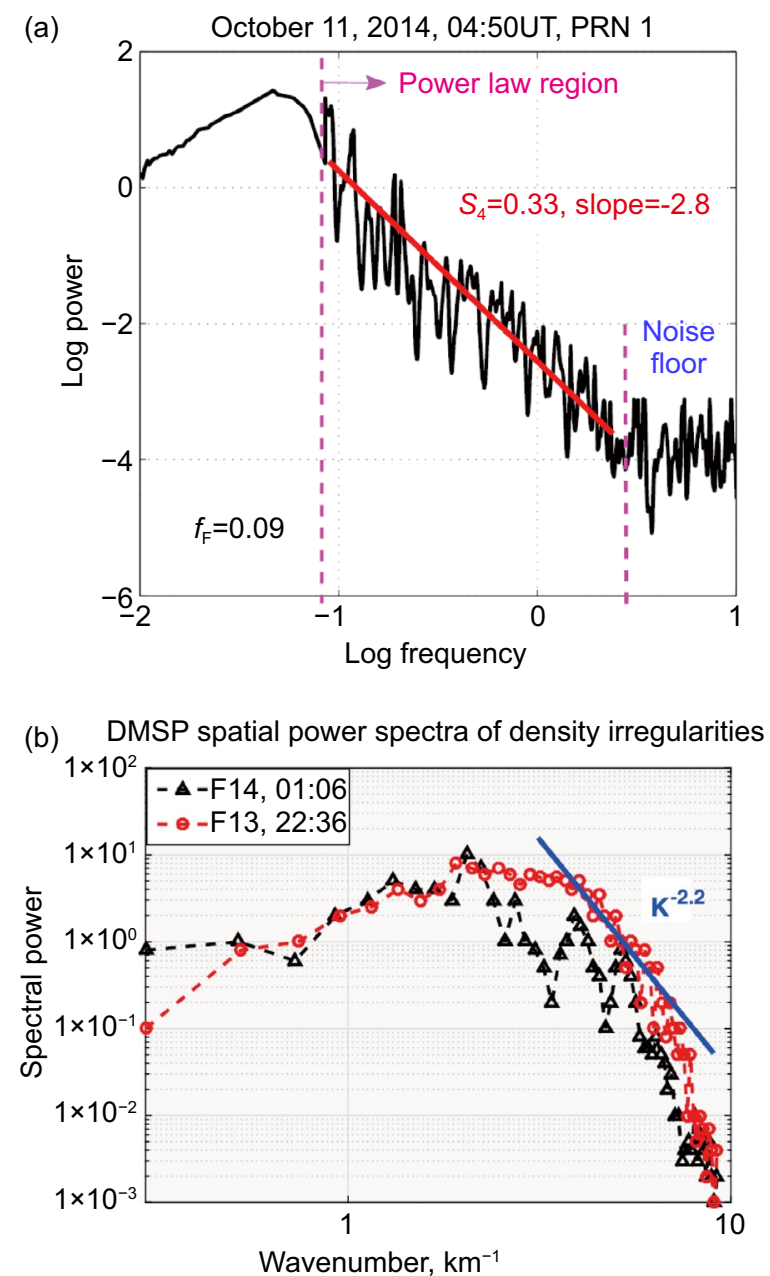

Fig. 10 a Power spectral of midlatitude GPS amplitude scintillation in October 2014. Spectal index $p=-2.8$ and fresnel scale is $0.09 \mathrm{~Hz}$. b Power spectral of in situ density irregularities observed by DMSP satellites F13 and F14 observed in September 2001 during a period of GPS scintillations. The spectral index of -2.2 is observed (Adapted from Eltrass et al. (2016))

from a spacecraft in ionospheric source regions of GNSS scintillation. Figure 10a shows the spectrum of GNSS scintillations during the periods of moderate mid-latitude GNSS scintillations (Eltrass et al. 2016). The power spectral index of the scintillation indicates an equivalent index of approximately $f^{-2.8}$ and Fresnel scale of $0.09 \mathrm{~Hz}$. An important assumption on the stationarity of irregularities implies frequency and wavenumber spectra are equivalent. Figure 10b shows a typical irregularity power spectrum from the Defense Meteorological Satellite Program (DMSP) satellites F14 and F13 (Mishin and Blaunstein 2008) which is taken in a source region of GPS scintillations. Figure 10a shows a power spectral index of $k^{-2.2 \pm 0.2}$. It should 
be noted that this in situ spectrum will be compared with a local irregularity spectrum from the simulations of Eltrass and Scales (2014). There is relatively good agreement between the in situ power spectral characteristics and the local plasma turbulence simulations of the TGI. Although the spectral characteristics are in line between the plasma simulations and in situ DMSP measurements, which provides some evidence of the TGI as a generation mechanism of the turbulence, there is some discrepancy with the spectral characteristics of the GPS scintillations. However, as discussed in Eltrass et al. (2016), care must be taken to compare the GPS scintillation spectral characteristics with the satellite observations due to the nonlinear transformation because of the propagation effects through the irregularities and space. A rough adjustment of these effects implies that the GPS scintillation spectral index $p=2.8$ should be adjusted for the in situ index $n$ with the rough expression $n=p-1=1.8$ which puts the GPS scintillation spectral index closer to the in situ measurements and plasma simulations. Nonetheless, these initial results indicate that the TGI may be responsible for GNSS scintillations as predicted by Kintner et al. (2007) as well as simultaneous SuperDARN radar scatter. This work motivates further study of simultaneous SuperDARN radar and GNSS scintillation correlation, as well as further consideration of the TGI as a mechanism for GNSS scintillation at mid-latitudes. It should be noted that the work discussed was under quiet geomagnetic conditions, $\mathrm{Kp}<2$. It is important to note that circumstances of active geomagnetic conditions indicate the Gradient Drift Instability (GDI) may play a more important role in producing sub-auroral irregularities due to the stronger magnetospheric electric field under these conditions (Eltrass et al. 2014). Therefore, the future work should consider the possibility of growth and nonlinear evolution of both the GDI and TGI at mid-latitudes, the difference in spectral characteristics, and more comparisons to GPS scintillation power spectra to ultimately resolve the potential irregularity generation mechanisms for mid-latitude GNSS scintillations under a broader spectrum of geomagnetic conditions.

\section{A new space-based observation technique}

Ground-based ionospheric remote sensing using GNSS sensors have provided large amount of observation data that lead to many groundbreaking studies on ionospheric irregularities. However, ground-based observations are limited to certain regions of the Earth's atmosphere (ionosphere) that are mostly adjacent to continents and thus they have relatively poor vertical resolution on ionospheric irregularity structures. Space-based GNSS observation techniques do not have such geospatial limitations by utilizing spaceborne GNSS receivers on satellites, balloons, aerial vehicles, and other aerospace platforms for ionospheric radio soundings. In this section, we first overview a number of existing space-based GNSS observation techniques/missions, then introduce a newly proposed space-based GNSS observation technique by using satellite formation flying.

\section{Brief overview of existing space-based GNSS observation techniques}

Traditional space missions launch a single satellite that carries in-situ plasma sensors to directly measure ion and electron density. The role of GNSS receivers in space missions is typically a Positioning, Velocity, and Timing (PVT) tool to assist the satellite's on-board guidance, navigation, and control system. Conventionally, launching GNSS receivers into space (e.g., LEO) is primarily for engineering purposes instead of scientific measurements. In the past two decades, GNSS receivers also became a valuable space weather measurement tool in space missions. For example (Pinto Jayawardena et al. 2016), reconstructed global electron density profiles using ionospheric tomography techniques to process the GNSS data collected by a LEO satellite. The Constellation Observing System for Meteorology, Ionosphere, and Climate (COSMIC) mission uses the Radio Occultation (RO) technique (a bending effect on the GNSS signals propagating through the Earth's upper atmosphere) to measure space-based TEC and scintillations (Coster and Komjathy 2008).

In-situ plasma measurement techniques in single-satellite LEO missions [e.g., Langmuir probe on the China Seismo-Electromagnetic Satellite (Liu et al. 2019)], typically cannot provide the information with enough ThreeDimensional (3D) spatial resolution due to its single point measurement nature. Ionospheric tomography using LEO GNSS measurements can survey the 3D ionospheric electron density profiles. However, the temporal resolution is relatively poor using only the data from a single satellite. The fast temporal variation information of ionospheric irregularities can be missed out using such approaches. GPS RO technique, which was implemented by the COSMIC mission for example, cannot pinpoint the exact positions of ionospheric irregularities that cause GNSS scintillations or TEC gradients.

The European Space Agency (ESA) SWARM mission uses three LEO satellites to measure the Earth's magnetic field and ionosphere. As a passive formation flying mode, two of the three SWARM satellites are flying sideby-side. Because the SWARM satellite orbits are in close proximity to the ionosphere's $\mathrm{F}$ region, the in-situ plasma measurements plus the GNSS scintillation measurements 
from SWARM satellites can be effectively utilized to detect ionospheric irregularities from LEO (Xiong et al. 2016). The two SWARM satellites flying in formation, however, are not actively controlled in the sense to maneuver their relative orbits. So this type of satellite formation flying, also better known as satellite constellation operation, is not able to actively measure the ionospheric irregularities with a scale size of interest. A new spacebased GNSS observation technique using active satellite formation flying will be overviewed in the next section.

\section{GNSS-based spacecraft formation flying technique demonstration}

Spacecraft/Satellite Formation Flying (SFF) is a trending space mission concept which operates multiple satellites in proximity with each other as a team. Compared with traditional single-satellite missions, SFF engages a group of distributed space systems to work as a team. Launching a fleet of smaller satellites instead of a single large spacecraft can lower the mission cost. A satellite fleet in formation can be sustainable by replacing any team member satellite. From a scientific observation perspective, SFF enables multi-scale flexibility and robustness in terms of the volume, dimension, and resolution of geospace measurements.

SFF technologies can be seen in spacecraft development, rendezvous, docking (e.g., assembly of the International Space Station), and extravehicular activities (e.g., astronaut spacewalking). With a wide range of applications, a great number of satellite missions using SFF technologies were launched by NASA, ESA, German Aerospace Center (DLR), universities, and commercial companies in the last two decades. CLUSTER II is one of the pioneering SFF missions led by ESA (Escoubet et al. 2001). In July to August 2000, four similar CLUSTER II spacecraft were launched into highly elliptical polar orbits to study the Sun-Earth electromagnetic interactions by taking 3D in-situ measurements. Gravity Recovery And Climate Experiment (GRACE) is a joint mission of NASA and DLR using SFF with two spacecraft (Tapley et al. 2004). In March 2002, the GRACE satellites were launched into a Low Earth Orbit (LEO) with a default along-track separation of $220-\mathrm{km}$ to begin measuring the gravity field of the Earth. Launched in March 2015, Magnetospheric Multiscale Mission (MMS) is a NASA mission using SFF with a group of four satellites to predominantly survey the Earth's magnetosphere (Fuselier et al. 2016). The MMS satellites normally fly in tetrahedron, a pyramidshaped formation. In order to study the turbulence in the solar wind and the highly-dynamic magnetic reconnection process, the MMS satellites are operated with a strong flexibility to form another formation geometry.
Canadian Advanced Nanospace eXperiments 4 and 5 (CanX-4 and CanX-5), is a nanosatellite formation flying mission led by the University of Toronto and supported by Canadian Space Agency CANX4\&5. A pair of CubeSats $(15 \mathrm{~kg}, 20 \mathrm{~cm} \times 20 \mathrm{~cm} \times 20 \mathrm{~cm}$ each), CanX-4 and CanX-5, were launched into LEO in June 2014 to perform Along-Track Orbit (ATO) and Projected Circular Orbit (PCO) formation flight for technology demonstration purposes. HawkEye 360 Inc. is a pioneering company developing a formation flying satellite constellation for space-based Radio Frequency (RF) analytics HAWKEye360. StartRocket, a Russian startup company, plans to launch a cluster of CubeSats in formation and form space advertisement billboards by reflecting sunlight in the night sky to the Earth (StartRocket, https://startrocket.me/).

The robustness of SFF missions relies on the stable performance of the Guidance, Navigation and Control (GNC) system of each satellite. A realistic simulation platform that can test the functionalities of all the hardware and software systems relevant to the GNC system (e.g., space environment, spacecraft navigation sensor(s), onboard GNC software, spacecraft propulsion systems) is advantageous to validate the performance and demonstrate the mission feasibility before launch. For GNSSbased SFF missions, building a Hardware-in-the-Loop (HIL) simulation testbed including RF hardware GNSS simulators, GNSS receivers, communication systems and core GNC software system(s) is pivotal to prototype SFF missions. The first GNSS-based HIL simulation testbed for SFF, called the Formation Flying TestBed (FFTB), was developed at the NASA GSFC as reported in 2001 by Leitner (2001). The FFTB was used for the development, validation, and support of a number of SFF missions (e.g., MMS, University NanoSats (Hall et al. 1999), TechSat21 Burns et al. 2000). As a collaboration among NASA GFSC, DLR, and Universitv of Texas at Austin, the first results of real-time autonomously controlled closed-loop SFF simulations using the NASA FFTB were presented and demonstrated by Gill, Naasz and Ebinuma (2003). In 2004, the NASA FFTB was upgraded and implemented to simulate new HIL GPS-based SFF scenarios by Burns et al. (2004). At DLR, a GPS-based HIL SFF simulation testbed was developed to simulate and evaluate the realtime performance of a new relative navigation system in LEO scenarios as reported in Leung and Montenbruck (2005). The DLR SFF testbed was later implemented in a test campaign by Yamamoto and D'Amico (2008) to verify the GNC flight software for the PRISMA spacecraft autonomous formation flight mission. Besides NASA and DLR, GNSS-based HIL SFF simulation testbeds were also developed at several universities as well to design GNC software and support SFF mission developments. For 
example these testbeds were developed at University of Calgary (Marji 2008), University of Toronto (to support the CanX-4 and CanX-5 mission) (Eyer 2009), Yonsei University (Park et al. 2010), and more recently Stanford University (Giralo and D'Amico 2018).

Using Spirent hardware RF GNSS simulators, (Kowalchuk 2007) developed the Distributed Spacecraft Attitude Control System Simulator (DSACSS) testbed for spacecraft flight controller design, which was the initial attempt at Virginia Tech (VT) toward the establishment of a GNSS-based HIL simulation testbed for SFF. Later in 2016, (Harris 2016) further closed the gap toward the first prototype of GNSS-based SFF closed-loop simulation testbed at VT by establishing a GPS-based HIL testbed to simulate scenarios of ground vehicle, aircraft and LEO satellites with formation and tracking control algorithms. The development of GNSS-based SFF simulation testbeds greatly benefit the design of onboard GNC algorithms and several SFF missions. However, no previous testbed was directly developed for ionospheric space weather study. The Virginia Tech Formation Flying Testbed (VTFFTB), a HIL simulation testbed based on multiconstellation and multi-band GNSS, has been developed

Table $1 \mathrm{KPI}$ table of four VTFFTB development phases (Iono = Ionosphere; $\mathrm{Ne}=$ electron density)

\begin{tabular}{lllll}
\hline Phase & Constellation & $\begin{array}{l}\text { Satellite } \\
\text { number }\end{array}$ & Formation orbit & $\begin{array}{l}\text { lono Ne } \\
\text { model }\end{array}$ \\
\hline 1 & GPS & 2 & Fixed relative state & 1D \\
2 & GPS\&GAL & 2 & Fixed relative state & 1D \\
3 & GPS\&GAL & 3 & Natural orbits & $1 \mathrm{D}$ \\
4 & GPS\&GAL & 3 & Natural orbits & $3 \mathrm{D}$ \\
\hline
\end{tabular}

recently to simulate real-time closed-loop LEO SFF scenarios with a group of two or three satellites (Peng et al. 2019; Peng and Scales 2019; Peng et al. 2020). As shown in Table 1, the development of VTFFTB can be overviewed in four different phases.

\section{VTFFTB development phase 1}

In phase 1, a GPS-based satellite formation flying HIL simulation testbed for a formation of two LEO spacecraft was established with onboard ionospheric remote sensing capability (Peng et al. 2020). The VTFFTB was successfully established for closed-loop HIL simulations of SFF. As shown in Fig. 11, the infrastructure includes a GPS RF hardware signal simulator, a multi-constellation and multi-band GNSS receiver, a navigation \& control system, an STK visualization system, and an ionospheric remote sensing system. In real-time SFF simulation scenarios, the GNSS receiver was used to track the multifrequency GPS signals from the GPS simulator. The navigation and control system is operated synchronously to extract GNSS data for real-time relative state estimation and formation orbit control. The real-time formation flight capability of VTFFTB has been validated by benchmarking with a reference test scenario in Park et al. (2010).

As shown in Fig. 12, a simplified Equatorial Spread-F (ESF) scenario was designed to demonstrate VTFFTB applications in ionospheric irregularity (e.g., Equatorial Plasma Bubble (EPB)) observations. The vertical electron density distribution was modelled globally using OneDimensional (1D) vertical TEC profiles. The S4 index inside the simulated ESF region (above the Jicamarca Radio Observatory (JRO)) was modelled as 0.4 . Using a

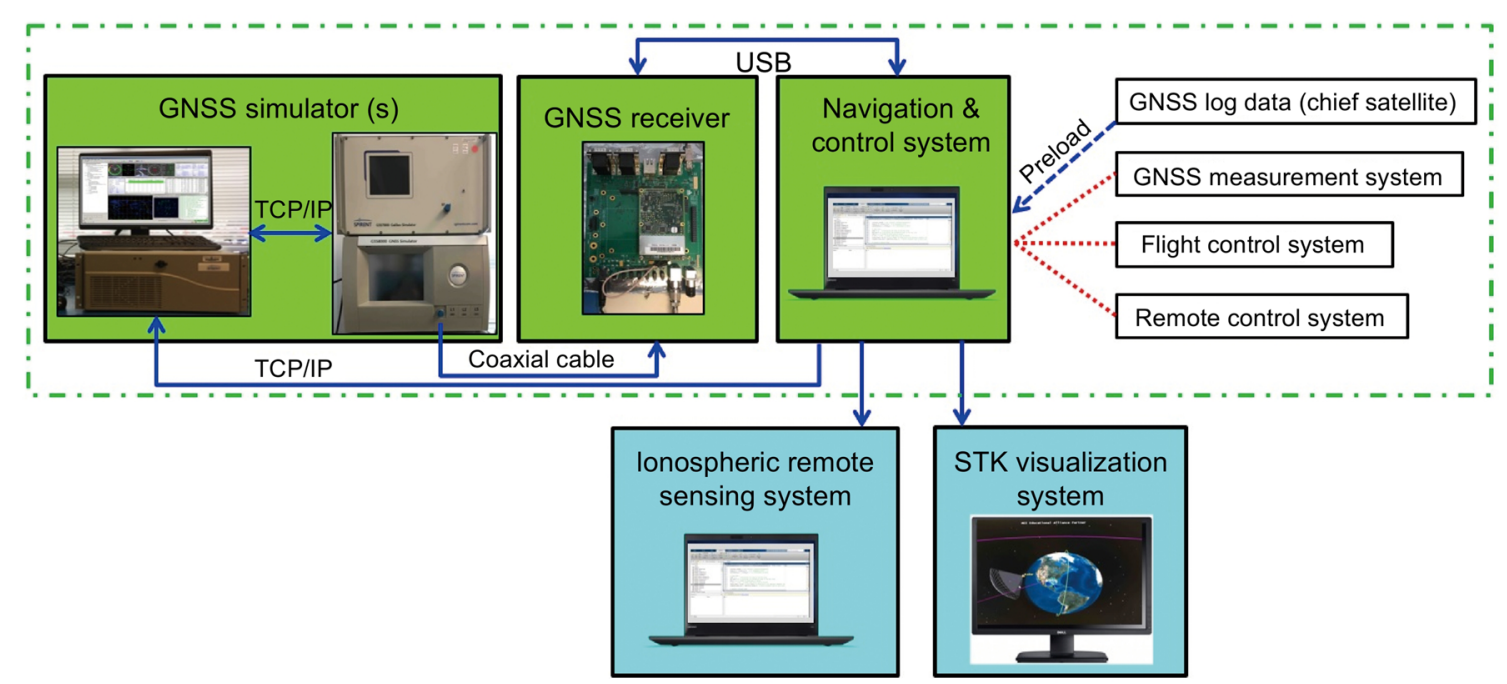

Fig. 11 GPS-based VTFFTB configuration. (Adapted from Peng et al. (2020)) 


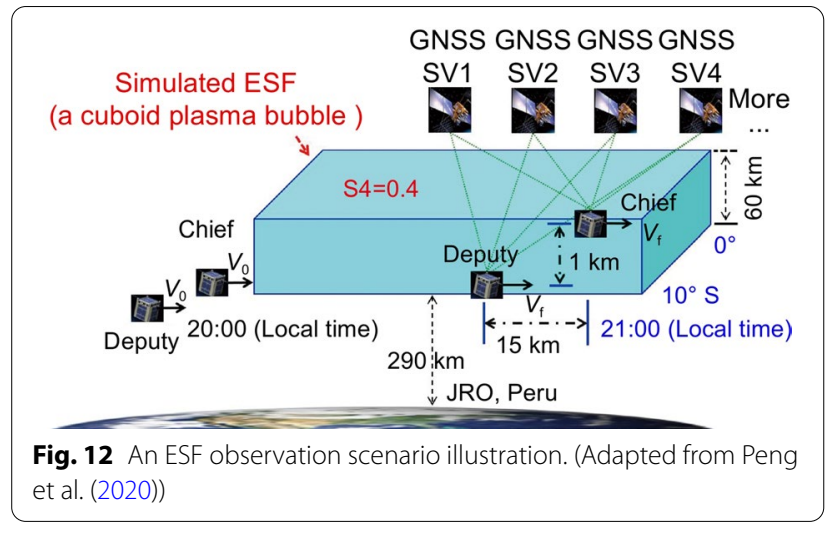

pair of LEO formation satellites, TEC and $C / N_{0}$ (carrierto-noise density) can be measured between GPS satellites and two LEO GNSS receivers in proximity. After each real-time formation flight simulation, the GPS navigation data was processed by the ionospheric remote sensing system to produce TEC, effective amplitude scintillation index (S4), and electron density (via differencing TEC). The electron density $(\mathrm{Ne})$ and $\mathrm{S} 4$ measurement results validated that using GPS-based SFF is able to retrieve the vertical Ne profile of an EPB event observed by the Langmuir probe on a Sounding Rocket (SR). An associated formation configuration sensitivity study shows that the VTFFTB can be used to optimize the Ne retrieval accuracy and precision by simulating various SFF orbits. The sensitivity study can lead one to believe that it will apply to other Ne profiles with different ionospheric structure or peak Ne value. Given a growing interest at VT to develop future SFF missions with potential space weather applications, establishing the first operational VTFFTB can incubate novel SFF missions in house and support test campaigns for ongoing and future ionospheric mission development.

\section{VTFFTB development phase 2}

If using GPS only is the "basic plan", utilizing multiple GNSS constellations can be regarded as a "premium plan" version of GPS to access a much wider variety of publicly available GNSS signals and a higher spatial converge of GNSS SV. In phase 2, the GPS-based VTFFTB was upgraded to a multi-constellation version adding the Galileo Navigation Satellite System (Galileo) constellation. Peng and Scales (2019) presented the VTFFTB development by adding Galileo and demonstrated the benefits of using multi-constellation GNSS for relative navigation and ionospheric remote sensing.

The ESF scenario proposed in stage 1 (Section VTFFTB development phase 1) was simulated again in the multi-constellation version VTFFTB. Ionospheric

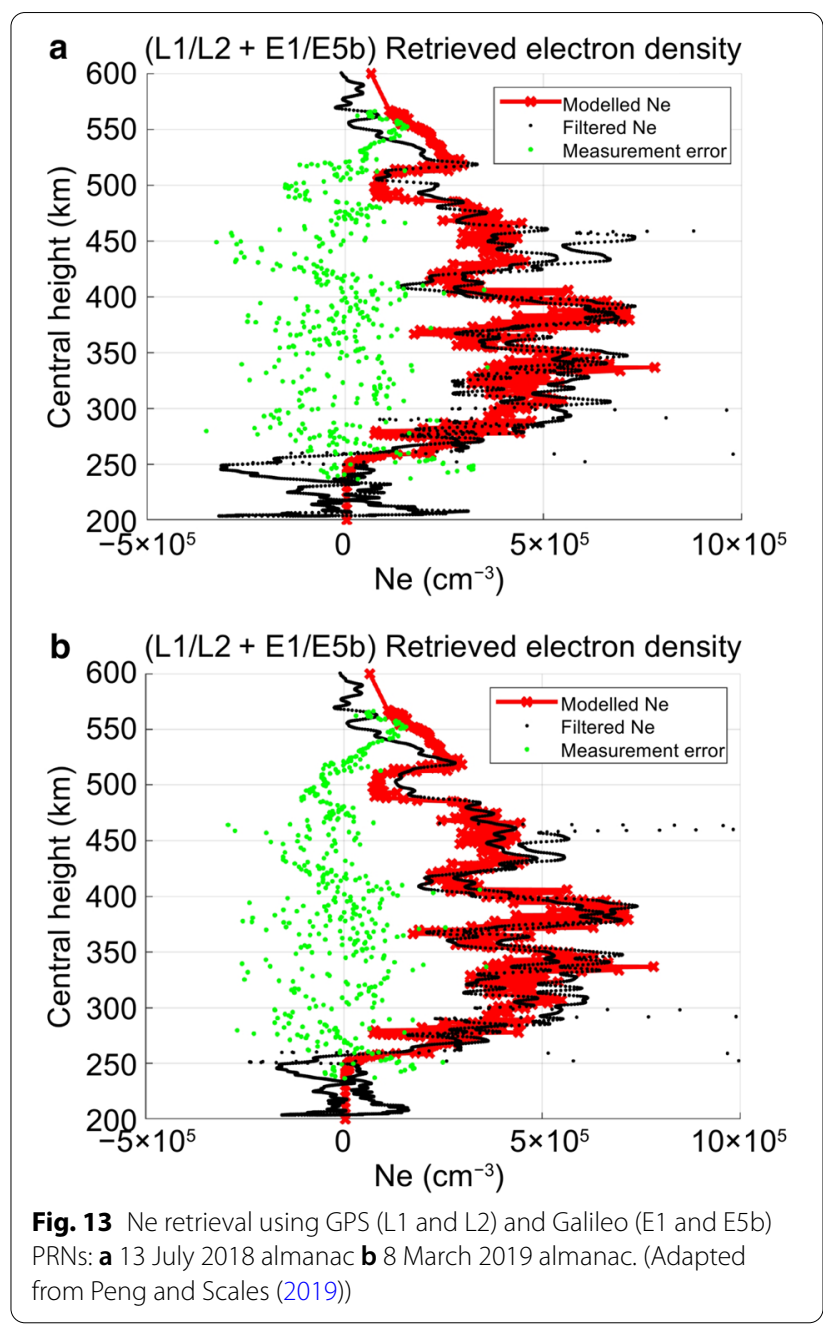

measurements (TEC, Ne, and effective S4) using both GPS and Galileo data were taken in HIL simulations. The cases of using L1 and L2 GPS signals were compared with the cases using dual-band multi-constellation (GPS and Galileo) signals. Compared with GPS only results, integrating GPS and Galileo measurements decreases the average and standard deviation of Ne retrieval errors by $32.83 \%$ and $46.12 \%$, respectively, in the scenario with the 13 July 2018 almanac as shown in Fig. 13a. As the Galileo constellation is still growing, HIL simulations can validate the modernization benefits with more and more Galileo SVs. A simulation using the 8 March 2019 almanac (22 operational Galileo SV) was done to compare with the 13 July 2018 version of Galileo (13 operational Galileo SV). The Ne retrieval result using the GPS and Galileo SVs on 8 March 2019 was plotted in Fig. 13b and the results show that using the more recent GNSS almanac further reduced the mean and standard deviation of Ne retrieval errors compared to Fig. 13a. 


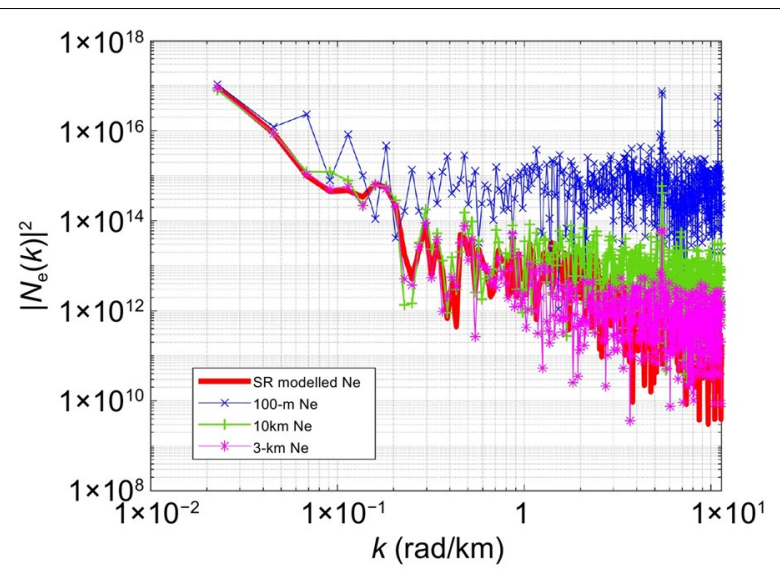

Fig. 14 Wavenumber spectrum comparison between different vertical separations. (Adapted from Peng and Scales (2019))

Similar to the cases in GPS simulation scenarios, Galileo Ne measurement accuracy and noise level are correlated to the vertical separation between the two GNSS receivers flying in formation as well. As shown in Fig. 14, an irregularity wavenumber spectrum analysis was undertaken to compare the Ne retrieval results between the configurations with three different vertical separations. The $100 \mathrm{~m}$ case exhibits the largest deviation across all the irregularity spatial range. Both the $1 \mathrm{~km}$ and $3 \mathrm{~km}$ separation cases agree well with the SR Ne model spectrum. Particularly, the smaller scale $(k>1)$ range agreement for the $1 \mathrm{~km}$ case is higher than for the $3 \mathrm{~km}$ case.
Besides satellite formation geometry, Ne measurements are found to be also correlated to the $\mathrm{C} / \mathrm{N}_{0}$ level of GNSS signals. Other than enhancing ionospheric measurement quality, adding Galileo for SFF also improved the performance of relative state estimation (higher accuracy and shorter convergence time) compared to using GPS only.

\section{VTFFTB development phase 3}

In phase 3, the 2-satellite setup of VTFFTB described in Section VTFFTB development phase 2 was further extended to a 3-satellite formation configuration as reported in Peng et al. (2019). Additional hardware equipment (e.g., GNSS RF signal simulators, GNSS receivers, and computers) were added and a new navigation \& control system was developed based on a decentralized approach of relative orbit estimation and control. The overall infrastructure is shown in Fig. 15. A fuel-efficient type of relative orbit (commonly known as natural orbit) was implemented to optimize the orbit configurations for small satellite formation flight. Three different formation modes (i.e., leader-follower, elliptic-orbit, and side-by-side) were chosen to evaluate the formation maneuverability for a group of three LEO satellites on the VTFFTB. A polar sun synchronous orbit scenario was designed and simulated to validate 3-satellite real-time formation flying capability of the updated VTFFTB.

A geo-space observation scenario using LEO SFF together with the AAL-PIP and SuperDARN networks was proposed in Peng et al. (2019) to address the studies similar to the discussion in Section Space weather

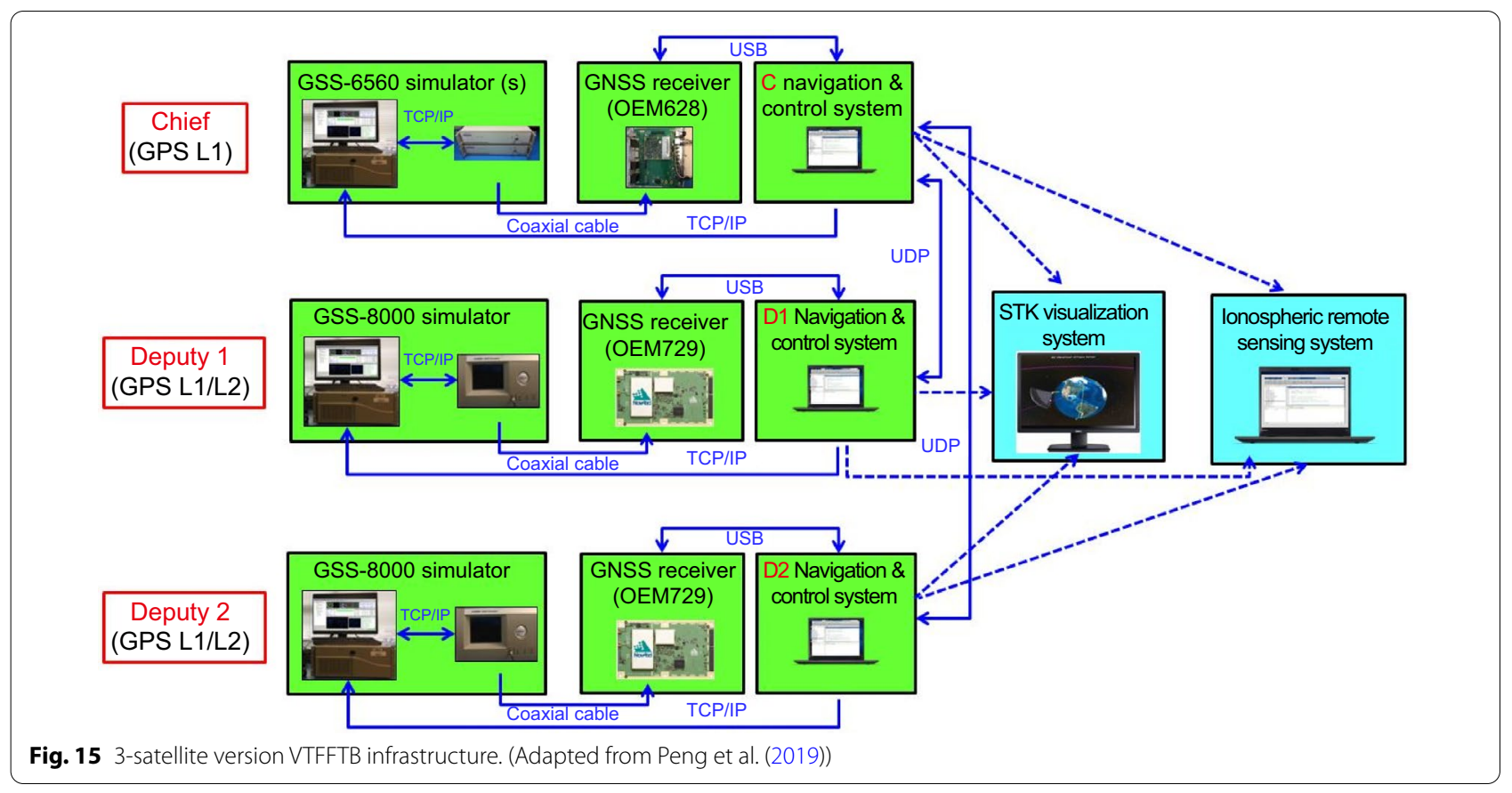


observations. This mission concept will be helpful to answer the following scientific questions: (1) How do ULF waves propagate in the south polar region? (2) How do ionospheric irregularities cause GNSS scintillations in high-latitude or polar regions? (3) How can these observations be used to better monitor geomagnetic storms and mitigate their impacts on GNSS and communication systems?

\section{VTFFTB development phase 4}

As there is a lack of fidelity of ionospheric modelling in phase 1 to phase 3 , the main focus in phase 4 is to enhance the fidelity of the ionospheric models used in the simulations performed on the VTFFTB (Peng 2020; Peng et al. 2021). The Thermosphere-Ionosphere-Electrodynamics-General-Circulation-Model (TIEGCM), a global ionospheric model developed by the U.S. National Center for Atmospheric Research (NCAR), was chosen to be integrated into the VTFFTB simulations. The global ionospheric electron density profiles from TIEGCM are used to simulate global ionospheric delay impacts on multi-band GNSS RF signals. As an example, the northern hemisphere electron density profiles at multiple TIEGCM height layers are shown in Fig. 16. As a snippet of the 2013 Saint Patrick's Day storm, the ionosphere at
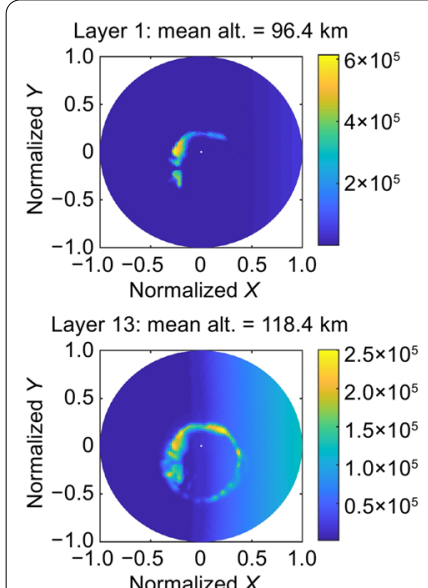

Layer 25: mean alt. $=192.8 \mathrm{~km}$

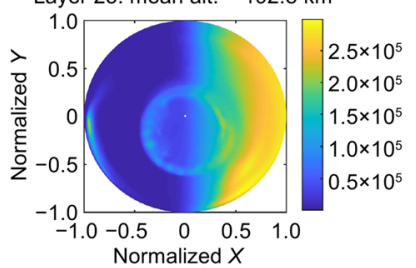

Layer 37 : mean alt. $=330.4 \mathrm{~km}$

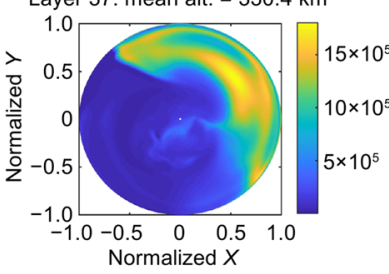

Layer 49: mean alt. $=517.3 \mathrm{~km}$

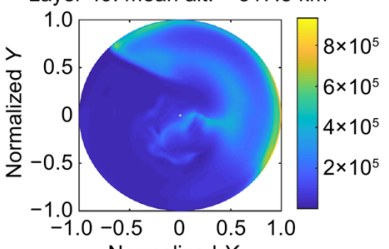

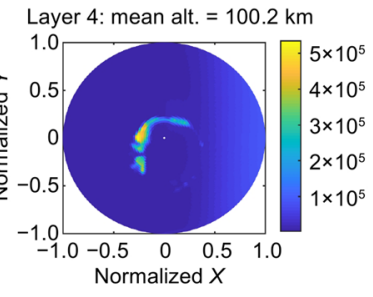

Layer 16: mean alt. $=131.0 \mathrm{~km}$

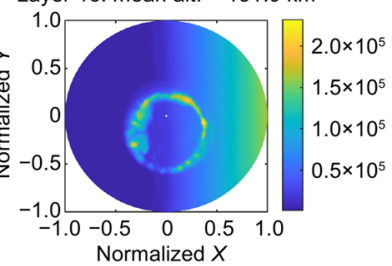

Layer 28: mean alt. $=221.5 \mathrm{~km}$

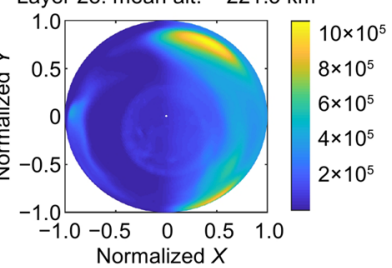

Layer 40: mean alt. $=373.4 \mathrm{~km}$

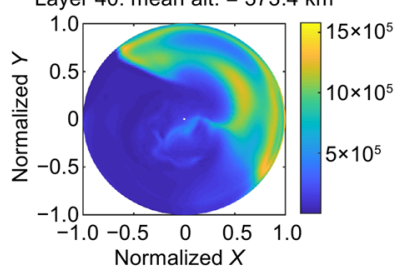

Layer 52: mean alt. $=569.1 \mathrm{~km}$

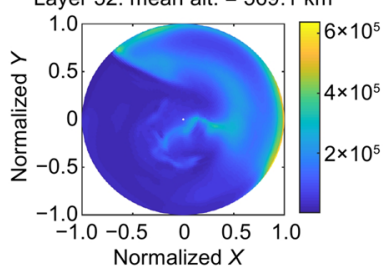

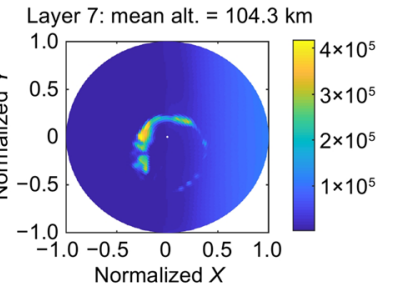

Layer 19: mean alt. $=147.7 \mathrm{~km}$
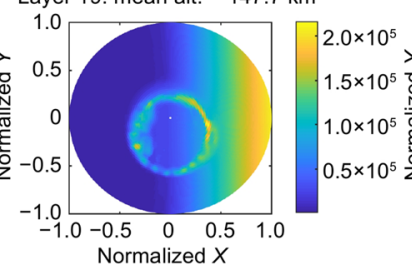

Layer 31: mean alt. $=254.1 \mathrm{~km}$
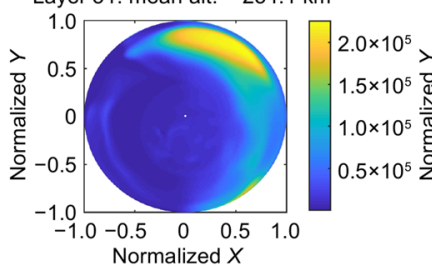

Layer 43: mean alt. $=419.1 \mathrm{~km}$
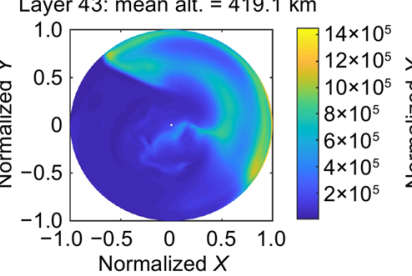

Layer 55: mean alt. $=622.3 \mathrm{~km}$

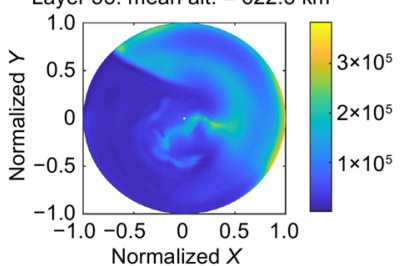

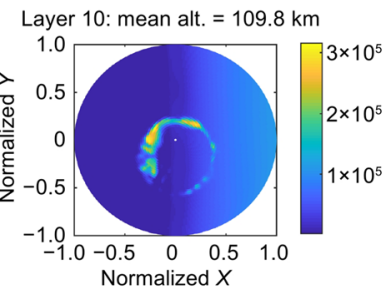

Layer 22: mean alt. $=168.3 \mathrm{~km}$

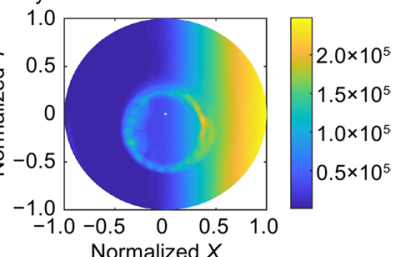

Layer 34: mean alt. $=290.5 \mathrm{~km}$

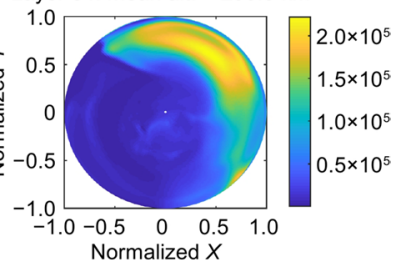

Layer 46: mean alt. $=467.2 \mathrm{~km}$

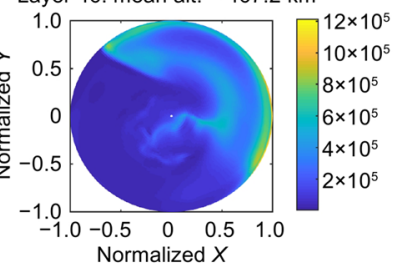

Normalized $X$

Fig. 16 Northern hemisphere's electron density profile at multiple TIEGCM height layers 12:00:00 UTC on March 17, 2013). (Adapted from Peng (2020)) 


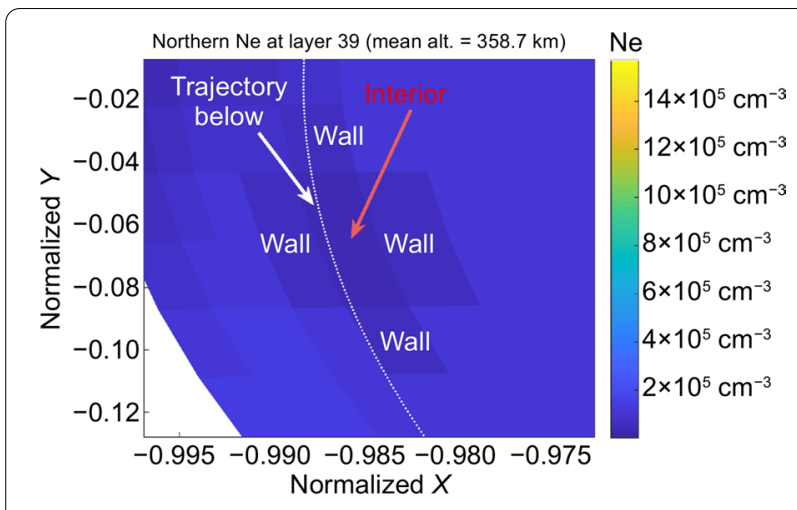

Fig. 17 A gnomonic projection view of LEO SFF trajectory passing the EPB. (Adapted from Peng and Scales (2020))

the time is extremely active and multi-scale ionospheric structures can be seen.

Using this TIEGCM background ionosphere, an EPB was added in a VTFFTB simulation to study the ionospheric irregularity impact on GNSS-based LEO formation flight (Peng and Scales 2020). As shown in Fig. 17, a pair of LEO satellites with GNSS receivers fly pass the $\mathrm{EPB}$ region along the white dotted line in two HIL simulations. The normalized $X$ and $Y$ are spherical coordinates at ground level transferred from geographical latitude and longitude. The shaded region with five cells (an interior and four walls) is a cross-section view of the added EPB. In the simulation when LEO receivers passed right below the EPB, a STEC trough can be clearly seen for each SV due to a GNSS signal interacting with the low ionospheric electron density region. In another simulation when LEO receivers flew through the EPB, an electron density profile of the EPB can be retrieved by the previously proposed differencing TEC method and the measurement result is consistent the TIEGCM "truth". Other global ionospheric or tropospheric models besides TIEGCM can be integrated with the VTFFTB to simulate more space weather phenomena and GNSS scenarios. New ionospheric irregularity observation techniques using GNSS can be validated, designed, or developed using the VTFFTB or other similar HIL simulation testbeds.

\section{Summary}

This paper reviews the recent advances in the multi-scale ionospheric irregularity studies at VT using groundbased and space-based GNSS observations. As descried in Section Passive measurements of high latitude ionospheric structure, four GNSS dual-band CASES receivers in the AAL-PIP were deployed along the $40^{\circ}$ magnetic meridian chain and utilized to collect the ground-based GPS data for years in Antarctica. The AAL-PIP system and its GPS receiver operation protocol were overviewed in Section System overview and GPS receiver operation. The GPS data retrieval and processing routine are automated as discussed in Section GPS data processing. A carrier-phase TEC stack plot software was developed to process the AAL-PIP GPS data inventory and detect ULF wave modulation. Studies of space weather impacts (high-latitude scintillations and global scale ULF waves) on AAL-PIP GPS signals were discussed in Section Space weather observations. Besides passive measurements, active measurements on high-latitude ionospheric structure produced by ground-based high power HF transmitters and the impact on GNSS signals were discussed in Section Active measurement of high-latitude ionospheric structure and impacts on GNSS. It was shown that Stimulated Electromagnetic Emissions (SEE) measurements made with ground-based HF receivers can be used to sense the decimeter scale ionospheric irregularities that are associated with GNSS phase scintillations. SuperDARN HF radars and GNSS receivers are also used to observe ionospheric irregularity effects on GPS at mid-latitude. An important implication is that larger hectometer scale ionospheric irregularities typically associated with GNSS amplitude scintillations may nonlinearly cascade down to decameter scales and be sensed by HF space weather radars. The associated modelling and observation work are reported in Section Modelling and observation of mid-latitude ionospheric irregularity effects on GNSS.

Other than ground-based GNSS observations, spacebased GNSS measurement techniques and missions are reviewed in Section Brief overview of existing spacebased GNSS observation techniques. A new space-based GNSS observation concept using SFF is demonstrated in Section GNSS-based space-craft formation flying technique demonstration. The development of the VTFFTB and its applications to multi-scale ionospheric irregularity remote sensing is presented in four different phases: Section VTFFTB development phase 1, namely, establishing the original GPS version VTFFTB for dual-satellite formation flight; Section VTFFTB development phase 2, namely, upgrading to a multi-constellation (GPS and Galileo) version; Section VTFFTB development phase 3, namely, expanding to a 3-satellite version with orbit optimization; Section VTFFTB development phase 4, namely, enhancing ionospheric simulation capability using the TIEGCM global ionospheric model. The development of VTFFTB creates a versatile mission incubator for future multi-scale ionospheric irregularity measurement techniques. Using both ground-based and spacebased GNSS associated techniques (e.g., GNSS receivers, magnetometers, radars, HF receivers, SFF), multiple spatial and temporal scales of ionospheric irregularities 
in different regions are better observed and understood. This ultimately can improve the performance or robustness of satellite navigation (e.g., GNSS) and other related technologies (e.g., satellite communication).

\section{Acknowledgements}

The authors would like thank all the Virginia Tech faculty and students that have participated in GNSS research over the past decade for their important contributions to this work.

\section{Author's contributions}

Conceptualization: YP and WAS; Methodology: YP and WAS; Writing original draft: YP and WAS; Editing:YP, WAS, MDH, XZ, and SC; Review: YP, WAS, MDH, and $X Z$. All authors read and approved the final manuscript.

\section{Funding Information}

The VTFFTB was supported by the AFOSR (Grant No. 13-0658-09) and Virginia Tech. Z. Xu and M.D. Hartinger were supported by NSF-1543364 (Polar Experiment Network for Geospace Upper-atmosphere Investigations - PENGUIn: Interhemispheric Investigations along the 40-degree Magnetic Meridian) and 1744828 (Collaborative Proposal: A High-Latitude Conjugate Area Array Experiment to Investigate Solar Wind - Magnetosphere - lonosphere Coupling).

\section{Availability of data and materials}

Data sharing is not applicable to this article as no dataset was generated in this review article.

\section{Declarations}

\section{Competing interests}

The authors declare that they have no competing interests.

\section{Author details}

${ }^{1}$ Center for Space Science and Engineering Research, Virginia Tech, Blacksburg, VA 24061, USA. ${ }^{2}$ Bradley Department of Electrical and Computer Engineering, Virginia Tech, Blacksburg, VA 24061, USA. ${ }^{3}$ Space Science Institute, Boulder, CO 80301, USA. ${ }^{4}$ National Institute of Aerospace, Hampton, VA 23666, USA. ${ }^{5}$ Center for Space Science and Engineering Research, Virginia Tech, Blacksburg, VA 24061, USA.

Received: 17 December 2020 Accepted: 29 March 2021

Published online: 12 July 2021

\section{References}

ASTRA: CASES Receiver Documentation. http://cases.astraspace.net/docum entation/. Accessed 16 August 2020.

ASTRA: CASES Receiver Software. http://cases.astraspace.net/software.html. Accessed 16 August 2020.

ASTRA: Official Website of ASTRA, LLC. http://www.astraspace.net/. Accessed 16 August 2020.

Burns, R., McLaughlin, C. A., Leitner, J., \& Martin, M. (2000). Techsat 21: Formation design, control, and simulation. In Proceedings of 2000 IEEE Aerospace Conference (Cat. No. 00TH8484) (vol. 7, pp. 19-25). IEEE.

Burns, R., Naasz, B., Gaylor, D., \& Higinbotham, J. (2004). An environment for hardware-in-the-loop formation navigation and control. In AIAAAAS astrodynamics specialist conference and exhibit (p. 4735).

CANX4\&5: CANX-4 \& CANX-5: Mission (xxxx). http://www.utias-sfl.net/?page_ id $=410$. Accessed 24 November 2020.

Clauer, C., Kim, H., Deshpande, K., Xu, Z., Weimer, D., Musko, S., et al. (2014). An autonomous adaptive low-power instrument platform (aal-pip) for remote high-latitude geospace data collection. Geoscientific Instrumentation, Methods and Data Systems, 3(2), 211-227.

Coster, A., \& Komjathy, A. (2008). Space weather and the global positioning system. Space Weather, 6(6), 11.

de Larquier, S., Eltrass, A., Mahmoudian, A., Ruohoniemi, J., Baker, J., Scales, W., et al. (2014). Investigation of the temperature gradient instability as the source of midlatitude quiet time decameter-scale ionospheric irregularities: Part 1. Observations. Journal of Geophysical Research Space Physics, 119(6), 4872-4881.

Deshpande, K., Bust, G. S., Clauer, C. R., Rino, C. L., \& Carrano, C. S. (2014). Satellitebeacon ionospheric-scintillation global model of the upper atmosphere (sigma) i: High latitude sensitivity study of the model parameters. Journal of Geophysical Research: Space Physics, 119, 4026-4043. https://doi. org/10.1002/2013JA019699.

Deshpande, K. B. (2014). Investigation of high latitude ionospheric irregularities utilizing modeling and gps observations. Ph.D. thesis, Virginia Polytechnic Institute and State University.

Eltrass, A., \& Scales, W. (2014). Nonlinear evolution of the temperature gradient instability in the midlatitude ionosphere. Journal of Geophysical Research: Space Physics, 119(9), 7889-7901. https://doi.org/10.1002/2014JA020314.

Eltrass, A., Mahmoudian, A., Scales, W., de Larquier, S., Ruohoniemi, J., Baker, J., et al. (2014). Investigation of the temperature gradient instability as the source of midlatitude quiet time decameter-scale ionospheric irregularities: Part 2. Linear analysis. Journal of Geophysical Research Space Physics, 119(6), 4882-4893.

Eltrass, A., Scales, W., Erickson, P., Ruohoniemi, J., \& Baker, J. (2016). Investigation of the role of plasma wave cascading processes in the formation of midlatitude irregularities utilizing gps and radar observations. Radio Science, 51(6), 836-851.

Escoubet, C., Fehringer, M., \& Goldstein, M. (2001). The cluster mission-introduction. In Annales Geophysicae (vol. 19, pp. 1197-1200).

Eyer, J. (2009). A dynamics and control algorithm for low earth orbit precision formation flying satellites. Ph.D. thesis, University of Toronto.

Fejer, B. G., \& Kelley, M. (1980). Ionospheric irregularities. Reviews of Geophysics, 18(2), 401-454

Fuselier, S., Lewis, W., Schiff, C., Ergun, R., Burch, J., Petrinec, S., \& Trattner, K. (2016). Magnetospheric multiscale science mission profile and operations. Space Science Reviews, 199(1-4), 77-103.

Gaposchkin, E., \& Coster, A. J. (1993). Gps /1-12 bias determination. Massachusetts Institute of Technology Lincoln Laboratory: Technical report.

Gill, E., Naasz, B., \& Ebinuma, T. (2003). First results from a hardware-in-the-loop demonstration of closed-loop autonomous formation flying. In 26th Annual AAS guidance, navigation and control conference, Breckenridge, Colorado, USA.

Giralo, V., \& D'Amico, S. (2018). Distributed multi-gnss timing and localization for nanosatellites. In ION GNSS+, Miami, Florida, USA (pp. 2518-2534).

Greenwald, R., Baker, K., Dudeney, J., Pinnock, M., Jones, T., Thomas, E., et al. (1995). Darn/superdarn. Space Science Reviews, 71(1-4), 761-796.

Greenwald, R. A., Oksavik, K., Erickson, P. J. Lind, F. D., Ruohoniemi, J. M., Baker, J. B., \& Gjerloev, J. W. (2006). Identification of the temperature gradient instability as the source of decameter-scale ionospheric irregularities on plasmapause field lines. Geophysical Research Letters, 33(18), 129.

Gurevich, A. V. (2007). Nonlinear effects in the ionosphere. Physics-Uspekhi, 50(11), 1091

Gurevich, A., \& Zybin, K. (2006). Strong field aligned scattering of uhf radio waves in ionospheric modification. Physics Letters A, 358(2), 159-165.

Hall, C., Davis, N., DeLaRee, J., Scales, W., \& Stutzman, W. (1999). Virginia tech ionospheric scintillation measurement mission. In AIAA/Utah State University Conference on Small Satellites, Logan, UT, USA.

Harris Jr, F. B. (2016). Gnss hardware-in-the-loop formation and tracking control. MSc thesis, Virginia Tech.

Hartinger, M. D., Xu, Z., Clauer, C. R., Yu, Y., Weimer, D. R., Kim, H., et al. (2017). Associating ground magnetometer observations with current or voltage generators. Journal of Geophysical Research (Space Physics), 122(7), 7130-7141. https://doi.org/10.1002/2017JA024140.

HAWkEye360: Hawkeye 360 awards contract to build next-generation satellite constellation to achieve rapid revisit for global spectrum awareness. https://www.he360.com/hawkeye-360-awards-contract-to-build-nextgeneration-constellation/. Accessed 19 March 2020.

Hooke, W. H. (1968). lonospheric irregularities produced by internal atmospheric gravity waves. Journal of Atmospheric and Terrestrial Physics, 30(5), 795-823.

Huang, J., \& Kuo, S. (1994). A theoretical model for the broad upshifted maximum in the stimulated electromagnetic emission spectrum. Journal of Geophysical Research Space Physics, 99(A10), 19569-19576.

Hudson, M. K., \& Kelley, M. C. (1976). The temperature gradient drift instability at the equatorward edge of the ionospheric plasma trough. Journal of Geophysical Research, 81(22), 3913-3918. 
Jacobs, J., Kato, Y., Matsushita, S., \& Troitskaya, V. (1964). Classification of geomagnetic micropulsations. Journal of Geophysical Research, 69(1), $180-181$.

Kelley, M. C. (2009). The earth's ionosphere: Plasma physics and electrodynamics. New York: Academic press.

Kim, H., Cai, X., Clauer, C. R., Kunduri, R. B. S., Matzka, J., Stolle, C., \& Weimer, D. R. (2013). Geomagnetic response to solar wind dynamic pressure impulse events at high-latitude conjugate points. Journal of Geophysical Research (Space Physics), 118(10), 6055-6071.

Kim, H., Clauer, C. R., Deshpande, K., Lessard, M. R., Weatherwax, A. T., Bust, G. S., et al. (2014). Ionospheric irregularities during a substorm event: Observations of ULF pulsations and GPS scintillations. Journal of Atmospheric and Solar-Terrestrial Physics, 114, 1-8.

Kintner, P. M., Ledvina, B. M., \& De Paula, E. (2007). Gps and ionospheric scintillations. Space Weather, 5(9), 176.

Klobuchar, J. A. (1987). lonospheric time-delay algorithm for single-frequency gps users. IEEE Transactions on Aerospace and Electronic systems, 3, 325-331.

Kowalchuk, S. A. (2007). Investigation of nonlinear control strategies using gps simulator and spacecraft attitude control simulator. Ph.D. thesis, Virginia Tech.

Leitner, J. (2001). A hardware-in-the-loop testbed for spacecraft formation flying applications. In 2001 IEEE aerospace conference proceedings (Cat. No. 01TH8542), (vol. 2, pp. 2-615). IEEE.

Leung, S., \& Montenbruck, O. (2005). Real-time navigation of formation-flying spacecraft using global-positioning-system measurements. Journal of Guidance, Control, and Dynamics, 28(2), 226-235.

Leyser, T. (2001). Stimulated electromagnetic emissions by high-frequency electromagnetic pumping of the ionospheric plasma. Space Science Reviews, 98(3-4), 223-328.

Liu, C., Guan, Y., Zheng, X., Zhang, A., Piero, D., \& Sun, Y. (2019). The technology of space plasma in-situ measurement on the china seismo-electromagnetic satellite. Science China Technological Sciences, 62(5), 829-838.

Luo, X., Lou, Y., Xiao, Q., Gu, S., Chen, B., \& Liu, Z. (2018). Investigation of ionospheric scintillation effects on bds precise point positioning at lowlatitude regions. GPS Solutions, 22(63), 1-12.

Luo, X., Gu, S., Lou, Y., Chen, B., \& Song, W. (2020). Better thresholds and weights to improve gnss ppp under ionospheric scintillation activity at low latitudes. GPS Solutions, 24(17), 1-12.

Mahmoudian, A., Scales, W., Taylor, S., Morton, Y., Bernhardt, P., Briczinski, S., \& Ghader, S. (2018). Artificial ionospheric gps phase scintillation excited during high-power radiowave modulation of the ionosphere. Radio Science, 53(6), 775-789.

Marji, Q. (2008). Precise relative navigation for satellite formation flying using gps. MSc thesis, The University of Calgary.

Mikhailovskii, A. B. (1974). Theory of plasma instabilities: Instabilities of a homogeneous plasma. Instabilities of an inhomogeneous plasma (2nd ed.). New York: Consultants Bureau.

Milikh, G., Gurevich, A., Zybin, K., \& Secan, J. (2008). Perturbations of gps signals by the ionospheric irregularities generated due to hf-heating at triple of electron gyrofrequency. Geophysical Research Letters, 35(22), 1068.

Mishin, E., \& Blaunstein, N. (2008). Irregularities within subauroral polarization stream-related troughs and gps radio interference at midlatitudes. Midlatitude lonospheric Dynamics and Disturbances. Geophysical Monograph Series, 181, 291-295.

MIT: Madrigal CEDAR Database (xxxx). http://cedar.openmadrigal.org. Accessed 26 September 2020.

Najmi, A., Milikh, G., Secan, J., Chiang, K., Psiaki, M., Bernhardt, P., et al. (2014). Generation and detection of super small striations by $\mathrm{f}$ region $\mathrm{hf}$ heating. Journal of Geophysical Research Space Physics, 119(7), 6000-6011.

Najmi, A., Milikh, G., Yampolski, Y., Koloskov, A., Sopin, A., Zalizovski, A., et al. (2015). Studies of the ionospheric turbulence excited by the fourth gyroharmonic at haarp. Journal of Geophysical Research Space Physics, $120(8), 6646-6660$.

NASA: CDDIS (xxxx). ftp://cddis.nasa.gov/gnss/data/daily. Accessed 16 August 2020.

Park, J.-I., Park, H.-E., Park, S.-Y., \& Choi, K.-H. (2010). Hardware-in-the-loop simulations of gps-based navigation and control for satellite formation flying. Advances in Space Research, 46(11), 1451-1465.
Parkinson, B. W., Enge, P., Axelrad, P., \& Spilker Jr, J. J. (1996). Global positioning system: Theory and applications (Vol. II, pp. 510-511). American Institute of Aeronautics and Astronautics, Stanford, California.

Peng, Y. (2020). GNSS-based hardware-in-the-loop simulation of spacecraft formation flight: An incubator for future multi-scale ionospheric space weather studies. Ph.D. thesis, Virginia Tech.

Peng, Y., \& Scales, W. (2020). GNSS-based simulation of spacecraft formation flight: A case study of ionospheric plasma remote sensing. Radiation Effects and Defects in Solids, 175(11-12), 998-1001. https://doi.org/10. 1080/10420150.2020.1845689.

Peng, Y., \& Scales, W. A. (2019). Satellite formation flight simulation using multiconstellation GNSS and applications to ionospheric remote sensing. Remote Sensing, 11(23), 2851. https://doi.org/10.3390/rs11232851.

Peng, Y., Scales, W. A., \& Edwards, T. R. (2020). GPS-based satellite formation flight simulation and applications to ionospheric remote sensing. Navigation, 67(1), 3-21. https://doi.org/10.1002/navi.354.

Peng, Y., Scales, W. A., \& Lin, D. (2021). GNSS-based hardware-in-the-loop simulations of spacecraft formation flying with the global ionospheric model TIEGCM. GPS Solutions, 25, 65. https://doi.org/10.1007/ s10291-021-01099-X.

Peng, Y., Scales, W. A., Esswein, M. C., \& Hartinger, M. D. (2019). Small satellite formation flying simulation with multi-constellation GNSS and applications to future multi-scale space weather observations. In Proceedings of the ION GNSS+, Miami, FL, USA (pp. 2035-22047). https://doi.org/10. 33012/2019.16883

Pi, X., Mannucci, A., Lindqwister, U., \& Ho, C. (1997). Monitoring of global ionospheric irregularities using the worldwide gps network. Geophysical Research Letters, 24(18), 2283-2286.

Pilipenko, V., Belakhovsky, V., Murr, D., Fedorov, E., \& Engebretson, M. (2014). Modulation of total electron content by ulf pc5 waves. Journal of Geophysical Research Space Physics, 119(6), 4358-4369.

Pinto Jayawardena, T. S., Chartier, A. T., Spencer, P., \& Mitchell, C. N. (2016). Imaging the topside ionosphere and plasmasphere with ionospheric tomography using cosmic gps tec. Journal of Geophysical Research Space Physics, 121(1), 817-831.

Ribeiro, A., Ruohoniemi, J., Baker, J., Clausen, L., Greenwald, R., \& Lester, M. (2012). A survey of plasma irregularities as seen by the midlatitude blackstone superdarn radar. Journal of Geophysical Research Space Physics, 117(A2), 1397. https://doi.org/10.1029/2011JA017207.

Rideout, W., \& Coster, A. (2006). Automated GPS processing for global total electron content data. GPS Solutions, 10(3), 219-228. https://doi.org/10. 1007/s10291-006-0029-5.

Scales, W., Peng, Y., Sorenson, C., Hartinger, M., Coyle, S., Shi, X., Zou, S., Coppeans, T., Clauer, C. R., \& Xu, Z. (2019). Multi-station GPS TEC and magnetometer observations of ULF waves in antarctica. In AGU fall meeting 2019, San Francisco, CA, USA.

StartRocket: The Orbital Display by StartRocket. https://startrocket.me/. Accessed 19 March 2020.

Stewart, B. (1861). XXII on the great magnetic disturbance which extended from august 28 to september 7,1859 , as recorded by photography at the kew observatory. Philosophical Transactions of the Royal Society of London, $151,423-430$

Tapley, B. D., Bettadpur, S., Ries, J. C., Thompson, P. F., \& Watkins, M. M. (2004). Grace measurements of mass variability in the earth system. Science, 305(5683), 503-505.

Tripathi, V., \& Liu, C. (1993). O mode decay and upshifted electromagnetic emissions near cyclotron harmonics in the ionosphere. Journal of Geophysical Research Space Physics, 98(A2), 1719-1723.

Vierinen, J., Coster, A. J., Rideout, W. C., Erickson, P. J., \& Norberg, J. (2016). Statistical framework for estimating gnss bias. Atmospheric Measurement Techniques, 9(3), 1303-1312. https://doi.org/10.5194/amt-9-1303-2016.

Watson, C., Jayachandran, P., Singer, H. J., Redmon, R. J., \& Danskin, D. (2015). Large-amplitude gps tec variations associated with pc5-6 magnetic field variations observed on the ground and at geosynchronous orbit. Journal of Geophysical Research Space Physics, 120(9), 7798-7821.

Xi, H., \& Scales, W. (2001). Numerical simulation studies on the broad upshifted maximum of ionospheric stimulated electromagnetic emission. Journal of Geophysical Research Space Physics, 106(A7), 12787-12801. 
Xiong, C., Stolle, C., \& Lühr, H. (2016). The swarm satellite loss of gps signal and its relation to ionospheric plasma irregularities. Space Weather, 14(8), 563-577.

Xu, Z., Hartinger, M., Clauer, C., Peek, T., \& Behlke, R. (2017). A comparison of the ground magnetic responses during the 2013 and 2015st patrick's day geomagnetic storms. Journal of Geophysical Research Space Physics, 122(4), 4023-4036.

Xu, Z., Hartinger, M. D., Clauer, R., Weimer, D., Deshpande, K., Kim, H., et al. (2019). Newly established autonomous adaptive low-power instrument platform (AAL-PIP) chain on East Antarctic Plateau and operation. Advances in Polar Science, 3, 362-374. https://doi.org/10.13679/j.advps. 2019.0028.
Xu, Z., Hartinger, M., Oliveira, D. M., Coyle, S., Clauer, C., Weimer, D., \& Edwards, T. (2020). Interhemispheric asymmetries in the ground magnetic response to interplanetary shocks: The role of shock impact angle. Space Weather, 18(3), 2019-002427.

Yamamoto, T., \& D'Amico, S. (2008). Hardware-in-the-loop demonstration of gps-based autonomous formation flying. In Workshop on satellite navigation technologies (NAVITEC), ESTEC, Noordwijk, Netherlands.

\section{Publisher's note}

Springer Nature remains neutral with regard to jurisdictional claims in published maps and institutional affiliations.

\section{Submit your manuscript to a SpringerOpen ${ }^{\circ}$ journal and benefit from:}

- Convenient online submission

- Rigorous peer review

- Open access: articles freely available online

- High visibility within the field

- Retaining the copyright to your article

Submit your next manuscript at $\boldsymbol{\nabla}$ springeropen.com 